\title{
Group-Transfer Polymerization of Various Crotonates Using Organic Acid Catalysts
}

Yasumasa Takenaka* and Hideki Abe

Bioplastic Research Team, RIKEN Center for Sustainable Resource Science, 2-1 Hirosawa, Wako, Saitama 351-0198, Japan.

*E-mail: yasumasa.takenaka@riken.jp. 


\section{Contents}

\section{Page S3 - S6:}

I. Materials and reagents.

II. Instrumentation.

III. The group-transfer polymerization of alkyl crotonate.

IV. The radical polymerization of methyl crotonate and tert-butyl crotonate.

V. Analytical data of products.

\section{Pages S7 - S17:}

Figure S1 - S19.

\section{Pages S18:}

Scheme S1.

\section{Pages S19 - S27:}

Figure S20 - S37.

\section{Pages S28:}

Table S1. 


\section{Materials and reagents}

All manipulations were performed under a nitrogen atmosphere by using standard Schlenk techniques or an argon atmosphere in an MBraun LABmaster SP glovebox. Dichloromethane $\left(\mathrm{CH}_{2} \mathrm{Cl}_{2}\right)$ was obtained from Kanto Chemical Co., purified by an MBraun SPS-800 Solvent Purification System. Argon and Nitrogen were used after purification by passing through a Dryclean column (4^̊ molecular sieve) and a Gasclean CC-XR column purchased from Nikka Seiko. Methyl crotonate, ethyl crotonate, isopropyl crotonate, $n$-butyl crotonate, sec-butyl crotonate, isobutyl crotonate, tert-butyl crotonate, and $p$-tolyl crotonate were obtained from Tokyo Chemical Industry Co. and were used after distillation by calcium hydride. 1-Methoxy1-trimethylsiloxy-2-methyl-1-propene (MTS) was obtained from Sigma-Aldrich and was used after distillation by calcium hydride $\left(\mathrm{CaH}_{2}\right)$. Bis(trifluoromethanesulfonyl)amine $\left(\mathrm{HNTf}_{2}\right)$ and 2,8,9-triisobutyl-2,5,8,9-tetraaza-1-phosphabicyclo[3.3.3] undecane (TiBP) were obtained from Aldrich and were used without further purification. N(Trimethylsilyl)bis(trifluoromethanesulfonyl)imide $\quad\left(\mathrm{TMSNTf}_{2}\right)$ and 1[bis(trifluoromethanesulfonyl)methyl]-2,3,4,5,6-pentafluorobenzene $\quad\left(\mathrm{C}_{6} \mathrm{H}_{5} \mathrm{CHTf}_{2}\right) \quad$ were obtained from Tokyo Chemical Industry Co. and were used without further purification. Tris(trifluoromethanesulfonyl)methane ( $\left.\mathrm{HTf}_{3}\right)$ was obtained from SynQuest Labs and was used without further purification. $o$-Carorane and $m$-carborane were obtained from Wako Pure Chemical Industries and were used without further purification.

\section{Instrumentation}

All manipulations were performed under a dry and oxygen-free argon atmosphere using standard high vacuum Schlenk techniques or in a glovebox. ${ }^{1} \mathrm{H}$ NMR and ${ }^{13} \mathrm{C}$ NMR spectra were measured on a Varian NMR (500 MHz for ${ }^{1} \mathrm{H} \mathrm{NMR,} 125 \mathrm{MHz}$ for ${ }^{13} \mathrm{C}$ NMR) spectrometer. The molecular weights of the polymers were measured by size exclusion chromatography (SEC) on a Shimadzu GPC system equipped with a refractive index detecter RID-10A, Shodex GPC K-802 column, and Shodex GPC K-806M column using $\mathrm{CHCl}_{3}$ as eluent (the flow rate is $0.8 \mathrm{ml} / \mathrm{min}$ ) against polystyrene standards. A Matrix-Assisted Laser Desorption/Ionization Time-of-Flight mass spectroscopy analysis (MALDI-TOF-MS) was conducted using an ultrafleXtreme MALDI-TOF spectrometer (Bruker Daltonics, Billerica, MA) operating in the linear mode at the accelerating voltage of $15 \mathrm{kV}$. The glass temperature $\left(T_{\mathrm{g}}\right)$ of the polymers was measured through dynamic mechanical analysis (DMA), which was carried out on a Perkin 
Elmer DMA800 under a nitrogen atmosphere. Any thermal history difference of the polymers was eliminated by first heating the specimen to above $200{ }^{\circ} \mathrm{C}$, cooling at $10{ }^{\circ} \mathrm{C} / \mathrm{min}$ to $-50{ }^{\circ} \mathrm{C}$, and then recording the second DMA scan at $5{ }^{\circ} \mathrm{C} / \mathrm{min}$ from $-50{ }^{\circ} \mathrm{C}$ to $200{ }^{\circ} \mathrm{C}$. Thermal gravimetric analysis (TGA) of the polymers were carried out using a thermal analysis instrument (SII Nano Technology TG/DTA7200) from room temperature to $500{ }^{\circ} \mathrm{C}$ under nitrogen atmosphere at $10{ }^{\circ} \mathrm{C} / \mathrm{min}$.

\section{The group-transfer polymerization of ethyl crotonate at $-40^{\circ} \mathrm{C}$ for $12 \mathrm{~h}$}

A typical polymerization procedure was described as follow: ethyl crotonate $(\mathrm{EtCr})(5.71 \mathrm{~g}, 50$ mmol), MTS (87.2 mg, $0.50 \mathrm{mmol})$, and $\mathrm{CH}_{2} \mathrm{Cl}_{2}(20 \mathrm{~mL})$ were charged into a Schlenk tube in a glovebox. $\mathrm{A} \mathrm{CH}_{2} \mathrm{Cl}_{2}$ solution $(5 \mathrm{~mL})$ of $\mathrm{C}_{6} \mathrm{H}_{5} \mathrm{CHTf}_{2}(22.3 \mathrm{mg}, 0.050 \mathrm{mmol})$ was added to initiate the polymerization reaction. After a predetermined period of the reaction (for $12 \mathrm{~h}$ ), the reaction mixture was poured into methanol $(10 \mathrm{~mL})$, and then the solvents were removed by evaporation under reduced pressure. The residue was dissolved in $\mathrm{CHCl}_{3}$, and the solution was poured into a large amount of hexane. The precipitated polymer was collected by filtration, washed several times with hexane, and dried under vacuum for $24 \mathrm{~h}$. The polymerization of various alkyl crotonates was carried out in a similar manner.

\section{The radical polymerization of methyl crotonate and tert-butyl crotonate}

Into a glass pressure vessel $(30 \mathrm{~mL})$ were successively placed a Teflon-coated magnetic stir bar, methyl crotonate $(500 \mathrm{mg}, 5.0 \mathrm{mmol})$, tolunene $(10 \mathrm{~mL})$ and AIBN $(8.2 \mathrm{mg}, 0.050 \mathrm{mmol})$ or V$70 \mathrm{~L}(15.4 \mathrm{mg}, 0.050 \mathrm{mmol})$. The reaction mixture was stirred at $80{ }^{\circ} \mathrm{C}$ for 2 weeks. After quenched the polymerization by small amount of methanol, the solvent was removed. The radical polymerization of tert-butyl crotonate was carried out in a similar manner. The corresponding polymers were not given in these reactions.

\section{Analytical Data of Products}

\section{n-Propyl crotonate}

${ }^{1} \mathrm{H}$ NMR (500MHz, rt, $\left.\mathrm{CDCl}_{3}\right): \delta 0.96\left(t, J=7.4 \mathrm{~Hz}, 3 \mathrm{H}, \mathrm{CH}_{2} \mathrm{CH}_{3}\right), 1.68(d t, J=6.9 \mathrm{~Hz}$ and $\left.7.4 \mathrm{~Hz}, 2 \mathrm{H}, \mathrm{CH}_{2} \mathrm{CH}_{2} \mathrm{CH}_{3}\right), 1.88\left(d d, J=1.7 \mathrm{~Hz}\right.$ and $\left.6.9 \mathrm{~Hz}, 3 \mathrm{H}, \beta-\mathrm{CH}_{3}\right), 4.08(t, J=6.9 \mathrm{~Hz}, 2 \mathrm{H}$, 
$\left.\mathrm{OCH}_{2} \mathrm{CH}_{2}\right), 5.85(d q, J=1.7 \mathrm{~Hz}$ and $15.5 \mathrm{~Hz}, 1 \mathrm{H}, \mathrm{HC}=\mathrm{CHC}=\mathrm{O}), 6.97(d q, J=6.9 \mathrm{~Hz}$ and 15.5 $\left.\mathrm{Hz}, 1 \mathrm{H}, \mathrm{C}=\mathrm{CHCH}_{3}\right)$.

${ }^{13} \mathrm{C}$ NMR (125MHz, rt, $\left.\mathrm{CDCl}_{3}\right): \delta 10.4\left(\mathrm{CH}_{2} \mathrm{CH}_{3}\right), 17.9\left(\mathrm{CH}_{2} \mathrm{CH}_{2} \mathrm{CH}_{3}\right), 22.0\left(\beta-\mathrm{CH}_{3}\right), 65.7$ $\left(\mathrm{OCH}_{2} \mathrm{CH}_{2}\right), 122.8(\mathrm{HC}=C \mathrm{HC}=\mathrm{O}), 144.3\left(\mathrm{C}=\mathrm{CHCH}_{3}\right), 166.6(C=\mathrm{O})$.

\section{Poly(methyl crotonate)}

${ }^{1} \mathrm{H}$ NMR (500MHz, rt, $\left.\mathrm{CDCl}_{3}\right): \delta 0.81,0.85$ and $1.04\left(3 \mathrm{H}, \beta-\mathrm{CH}_{3}\right), 2.0-2.6(2 \mathrm{H}, \alpha-\mathrm{CH}$ and $\beta$ $\mathrm{CH}), 3.58$ and $3.63\left(3 \mathrm{H}, \mathrm{OCH}_{3}\right.$ and $\mathrm{OCH}_{3}$ of polymer end).

${ }^{13} \mathrm{C} \mathrm{NMR}\left(125 \mathrm{MHz}, \mathrm{rt}, \mathrm{CDCl}_{3}\right): \delta 10.5-13.4\left(\beta-\mathrm{CH}_{3}\right), 30.5-31.3$ and $33.21(\alpha-\mathrm{CH}), 51.14$ and $51.35\left(\mathrm{OCH}_{3}\right), 53.06(\beta-C \mathrm{H}), 173.3-175.0(C=\mathrm{O})$.

\section{Poly(ethyl crotonate)}

${ }^{1} \mathrm{H}$ NMR (500MHz, rt, $\left.\mathrm{CDCl}_{3}\right): \delta 0.85$ and $1.02\left(3 \mathrm{H}, \beta-\mathrm{CH}_{3}\right), 1.23\left(3 \mathrm{H}, \mathrm{CH}_{2} \mathrm{CH}_{3}\right), 2.0-2.6(2 \mathrm{H}$, $\alpha-\mathrm{CH}$ and $\beta-\mathrm{CH}), 3.62\left(\mathrm{OCH}_{3}\right.$ of polymer end), $4.06\left(2 \mathrm{H}, \mathrm{OCH}_{2}\right)$.

${ }^{13} \mathrm{C}$ NMR (125MHz, rt, $\left.\mathrm{CDCl}_{3}\right): \delta$ 10.5-14.0 $\left(\beta-\mathrm{CH}_{3}\right), 14.06\left(\mathrm{CH}_{2} \mathrm{CH}_{3}\right), 31.25$ and $33.40(\alpha-$ $C \mathrm{H}), 50.4-52.8(\beta-C \mathrm{H}), 59.75$ and $59.99\left(\mathrm{OCH}_{2}\right), 173.1-174.2(C=\mathrm{O})$.

\section{Poly(n-propyl crotonate)}

${ }^{1} \mathrm{H}$ NMR (500MHz, rt, $\left.\mathrm{CDCl}_{3}\right): \delta 0.83$ and $1.02\left(3 \mathrm{H}, \beta-\mathrm{CH}_{3}\right), 0.93\left(3 \mathrm{H}, \mathrm{CH}_{2} \mathrm{CH}_{3}\right), 1.61(2 \mathrm{H}$, $\left.\mathrm{CH}_{2} \mathrm{CH}_{2} \mathrm{CH}_{3}\right), 2.0-2.6(2 \mathrm{H}, \alpha-\mathrm{CH}$ and $\beta-\mathrm{CH}), 3.65\left(\mathrm{OCH}_{3}\right.$ of polymer end $), 3.93(2 \mathrm{H}$, $\left.\mathrm{OCH}_{2} \mathrm{CH}_{2}\right)$.

${ }^{13} \mathrm{C}$ NMR (125MHz, rt, $\left.\mathrm{CDCl}_{3}\right): \delta 10.29-10.41\left(\mathrm{CH}_{2} \mathrm{CH}_{3}\right), 10.3-14.5\left(\beta-\mathrm{CH}_{3}\right), 21.80$ and 21.92 $\left(\mathrm{CH}_{2} \mathrm{CH}_{3}\right), 31.24$ and $33.78(\alpha-\mathrm{CH}), 51.1-52.7(\beta-C \mathrm{H}), 65.56\left(\mathrm{OCH}_{2}\right), 172.8-174.5(C=\mathrm{O})$.

\section{Poly(n-butyl crotonate)}

${ }^{1} \mathrm{H}$ NMR (500MHz, rt, $\left.\mathrm{CDCl}_{3}\right): \delta 0.83$ and $1.01\left(3 \mathrm{H}, \beta-\mathrm{CH}_{3}\right), 0.92\left(3 \mathrm{H}, \mathrm{CH}_{2} \mathrm{CH}_{3}\right), 1.37(2 \mathrm{H}$, $\left.\mathrm{CH}_{2} \mathrm{CH}_{2} \mathrm{CH}_{3}\right), 1.58\left(2 \mathrm{H}, \mathrm{CH}_{2} \mathrm{CH}_{2} \mathrm{CH}_{2}\right), 2.0-2.6(2 \mathrm{H}, \alpha-\mathrm{CH}$ and $\beta-\mathrm{CH}), 3.63\left(\mathrm{OCH}_{3}\right.$ of polymer end), 3.95 and $4.01\left(2 \mathrm{H}, \mathrm{OCH}_{2} \mathrm{CH}_{2}\right)$.

${ }^{13} \mathrm{C}$ NMR (125MHz, rt, $\left.\mathrm{CDCl}_{3}\right): \delta$ 10.5-12.4 $\left(\beta-\mathrm{CH}_{3}\right), 13.69\left(\mathrm{CH}_{2} \mathrm{CH}_{3}\right), 19.06$ and 19.14 $\left(\mathrm{CH}_{2} \mathrm{CH}_{3}\right), 30.51\left(\mathrm{CH}_{2} \mathrm{CH}_{2} \mathrm{CH}_{2}\right), 30.59$ and $33.49(\alpha-\mathrm{CH}), 51.2-52.6(\beta-\mathrm{CH}), 63.78$ and 63.95 $\left(\mathrm{OCH}_{2} \mathrm{CH}_{2}\right), 172.8-174.5(C=\mathrm{O})$. 


\section{Poly(isopropyl crotonate)}

${ }^{1} \mathrm{H}$ NMR (500MHz, rt, $\left.\mathrm{CDCl}_{3}\right): \delta 0.86$ and $1.03\left(3 \mathrm{H}, \beta-\mathrm{CH}_{3}\right), 1.22\left(6 \mathrm{H}, \mathrm{CH}\left(\mathrm{CH}_{3}\right)_{2}\right), 2.0-2.6$ (2H, $\alpha-\mathrm{CH}$ and $\beta-\mathrm{CH}), 3.64\left(\mathrm{OCH}_{3}\right.$ of polymer end), $4.91\left(1 \mathrm{H}, \mathrm{OCH}\left(\mathrm{CH}_{3}\right)_{2}\right)$.

${ }^{13} \mathrm{C}$ NMR (125MHz, rt, $\left.\mathrm{CDCl}_{3}\right): \delta 11.0-13.2\left(\beta-\mathrm{CH}_{3}\right), 21.87\left(\mathrm{CH}\left(\mathrm{CH}_{3}\right)_{2}\right), 31.66(\alpha-C \mathrm{H}), 50.87$ $(\beta-\mathrm{CH}), 67.29\left(\mathrm{OCH}\left(\mathrm{CH}_{3}\right)_{2}\right), 172.8-174.5(C=\mathrm{O})$.

\section{Poly(isobutyl crotonate)}

${ }^{1} \mathrm{H}$ NMR (500MHz, rt, $\left.\mathrm{CDCl}_{3}\right): \delta 0.82$ and $1.02\left(3 \mathrm{H}, \beta-\mathrm{CH}_{3}\right), 0.92\left(6 \mathrm{H}, \mathrm{CH}\left(\mathrm{CH}_{3}\right)_{2}\right), 1.87(1 \mathrm{H}$, $\left.\mathrm{CH}\left(\mathrm{CH}_{3}\right)_{2}\right), 2.0-2.6(2 \mathrm{H}, \alpha-\mathrm{CH}$ and $\beta-\mathrm{CH}), 3.6-4.0\left(2 \mathrm{H}, \mathrm{OCH}_{2} \mathrm{CH}\right.$ and $\mathrm{OCH}_{3}$ of polymer end). ${ }^{13} \mathrm{C} \mathrm{NMR}\left(125 \mathrm{MHz}, \mathrm{rt}, \mathrm{CDCl}_{3}\right): \delta 10.5-13.5\left(\beta-\mathrm{CH}_{3}\right), 19.18$ and $19.33\left(\mathrm{CH}\left(\mathrm{CH}_{3}\right)_{2}\right), 27.50$ and $27.61\left(C \mathrm{H}\left(\mathrm{CH}_{3}\right)_{2}\right), 30.5-34.71(\alpha-C \mathrm{H}), 51.3-52.5(\beta-C \mathrm{H}), 70.18$ and $70.35\left(\mathrm{OCH}_{2} \mathrm{CH}\right), 172.7-$ $174.6(C=\mathrm{O})$.

\section{Poly(sec-butyl crotonate)}

${ }^{1} \mathrm{H}$ NMR (500MHz, rt, $\left.\mathrm{CDCl}_{3}\right): \delta$ 0.80-1.25 $\left(3 \mathrm{H}, \beta-\mathrm{CH}_{3}\right), 0.92\left(3 \mathrm{H}, \mathrm{CH}_{2} \mathrm{CH}_{3}\right), 1.21(3 \mathrm{H}$, $\left.\mathrm{CHCH}_{3}\right), 1.47$ and $1.66\left(2 \mathrm{H}, \mathrm{CH}_{2} \mathrm{CH}_{3}\right), 2.0-2.6(2 \mathrm{H}, \alpha-\mathrm{CH}$ and $\beta-\mathrm{CH}), 3.64\left(\mathrm{OCH}_{3}\right.$ of polymer end), $4.72(1 \mathrm{H}, \mathrm{OCH})$.

${ }^{13} \mathrm{C}$ NMR (125MHz, rt, $\left.\mathrm{CDCl}_{3}\right): \delta 9.83\left(\mathrm{CH}_{2} \mathrm{CH}_{3}\right), 11.17$ and 11.2-13.5 $\left(\beta-\mathrm{CH}_{3}\right), 18.78$ and $19.16\left(\mathrm{CHCH}_{3}\right), 28.69\left(\mathrm{CH}_{2} \mathrm{CH}_{3}\right), 31.72(\alpha-C \mathrm{H}), 50.73(\beta-C \mathrm{H}), 72.10(\mathrm{OCH}), 172.37(C=\mathrm{O})$. 


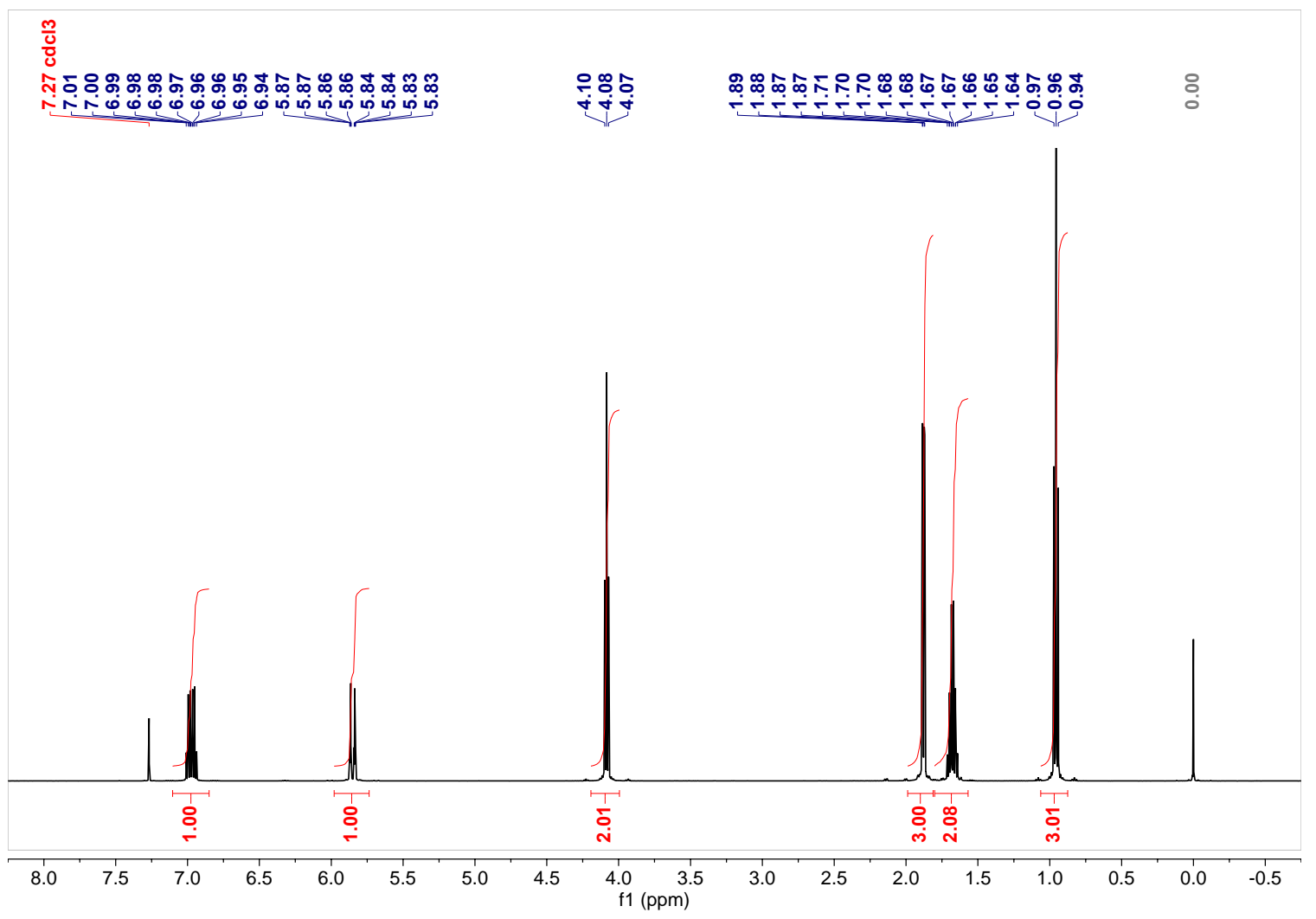

Figure S1. ${ }^{1} \mathrm{H}$ NMR spectrum of $n$-propyl crotonate

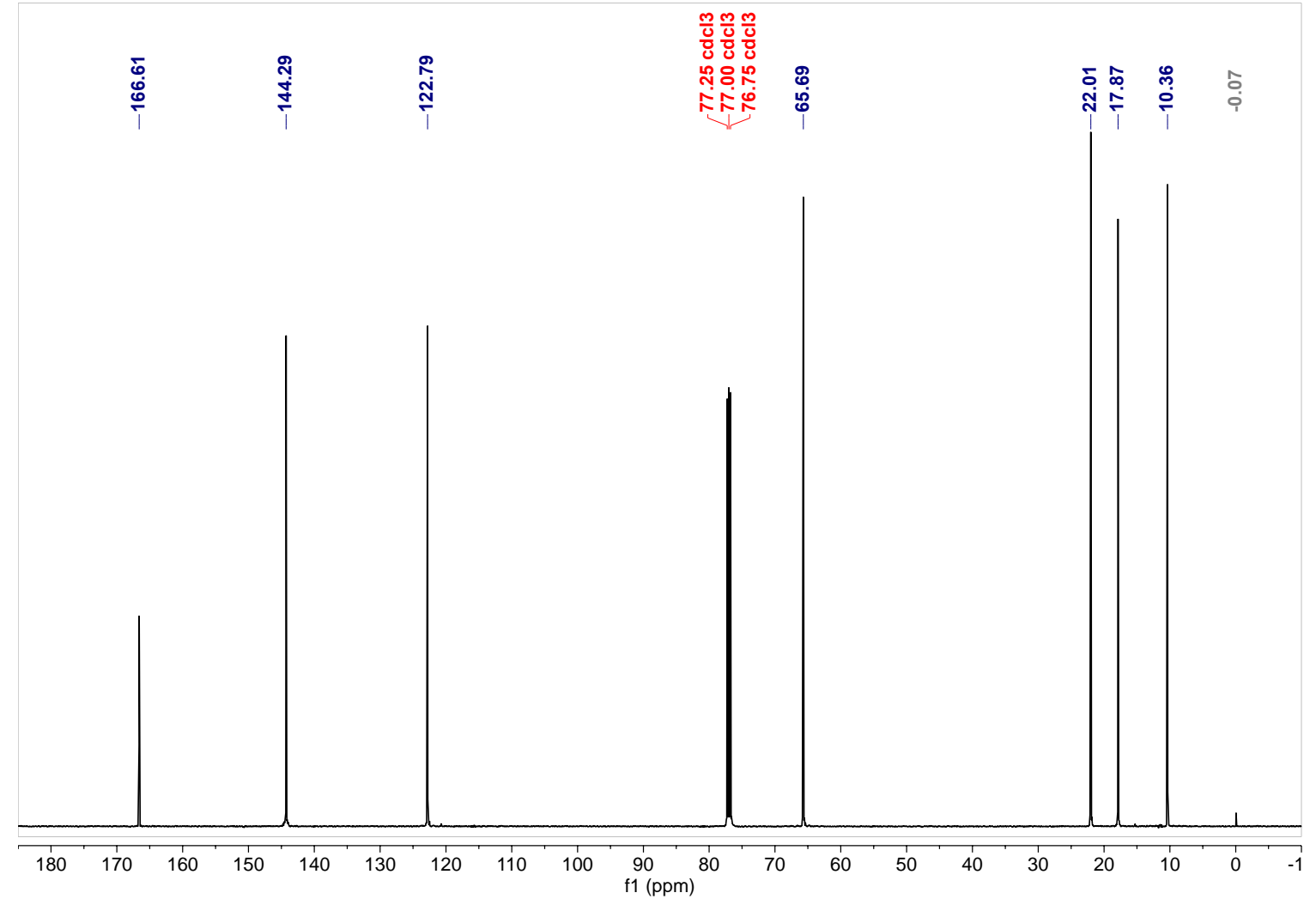

Figure S2. ${ }^{13} \mathrm{C}$ NMR spectrum of $n$-propyl crotonate 


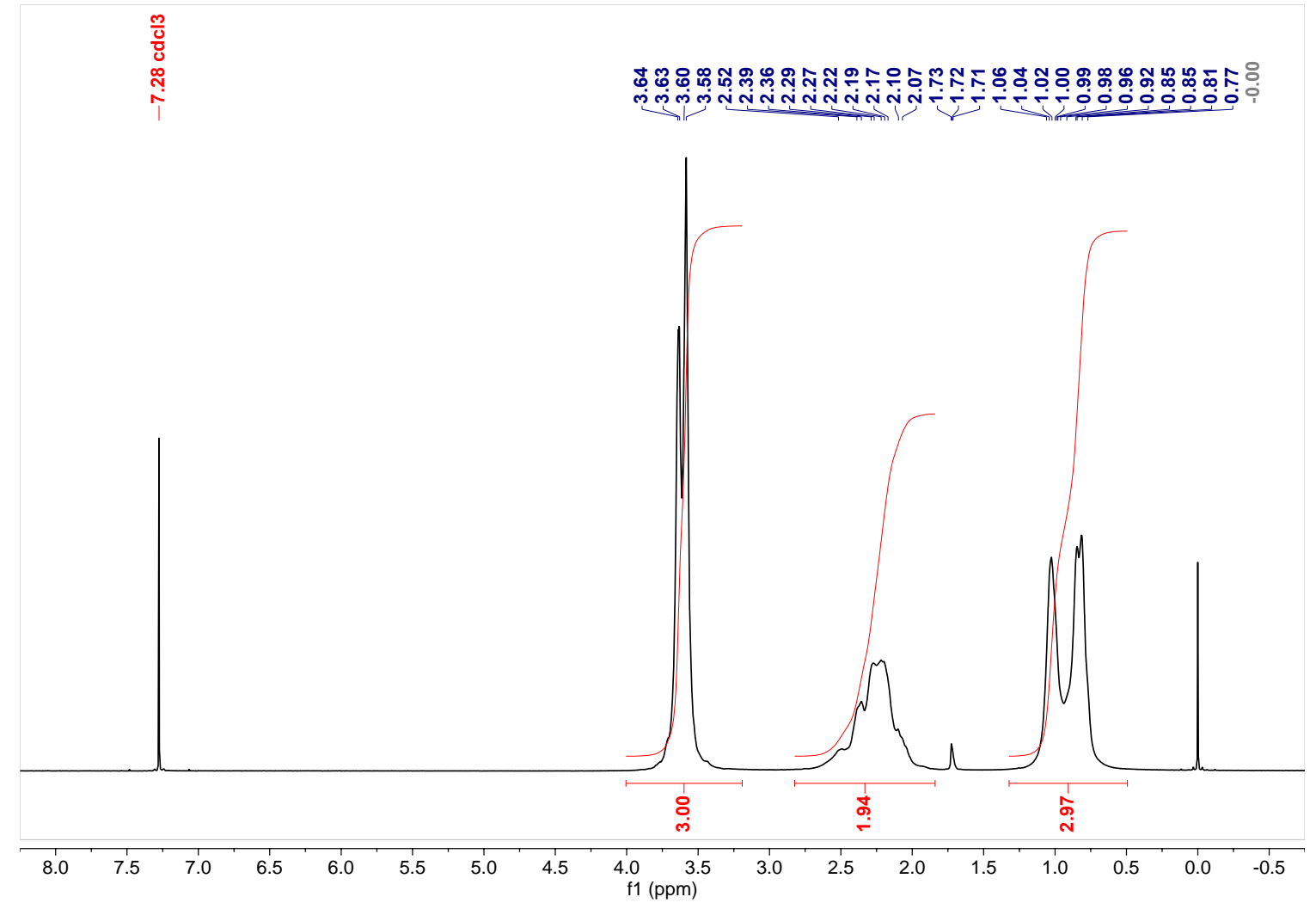

Figure S3. ${ }^{1}$ H NMR spectrum of poly(methyl crotonate) (Table 5, entry 27).

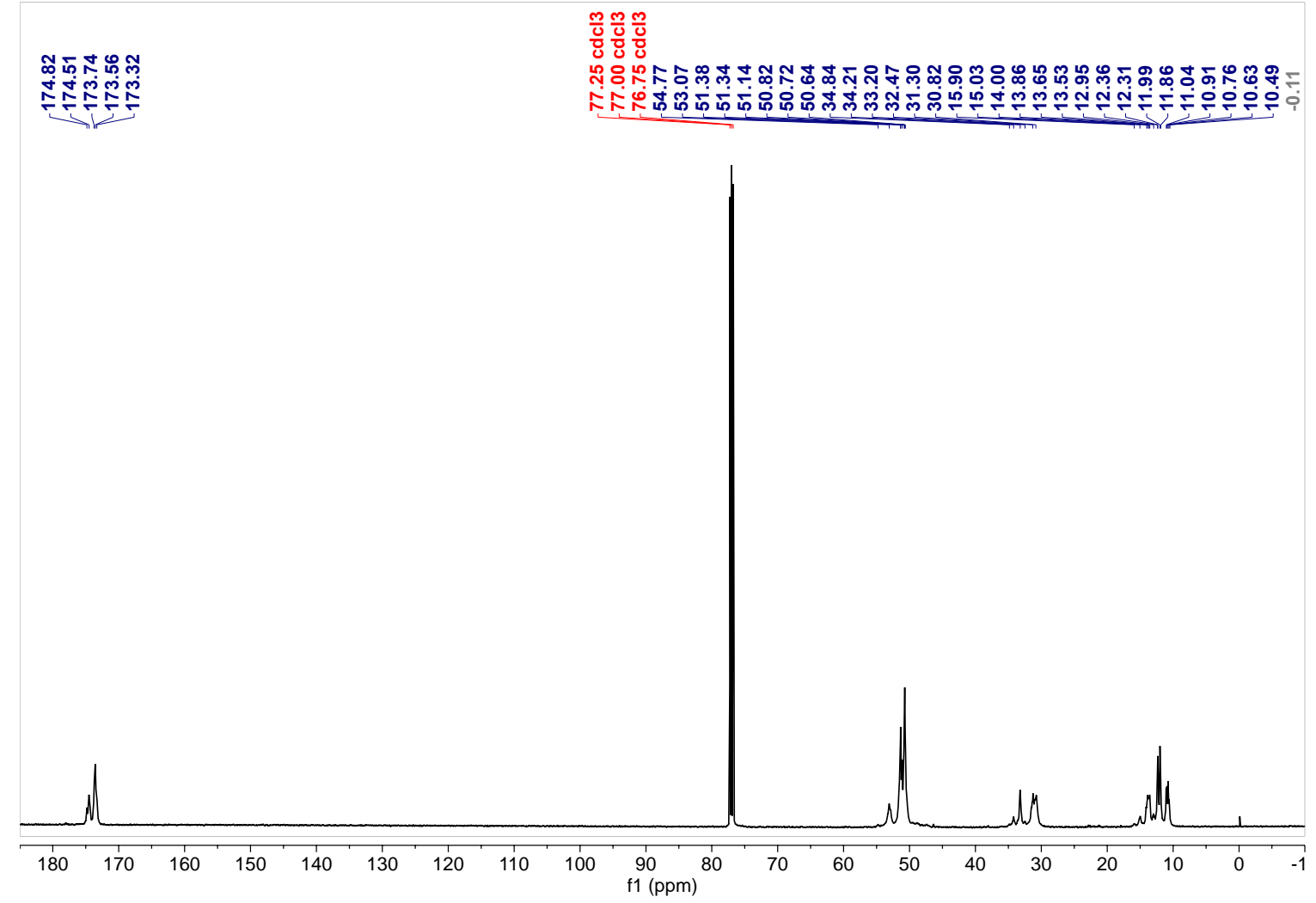

Figure S4. ${ }^{13} \mathrm{C}$ NMR spectrum of poly(methyl crotonate) (Table 5, entry 27). 


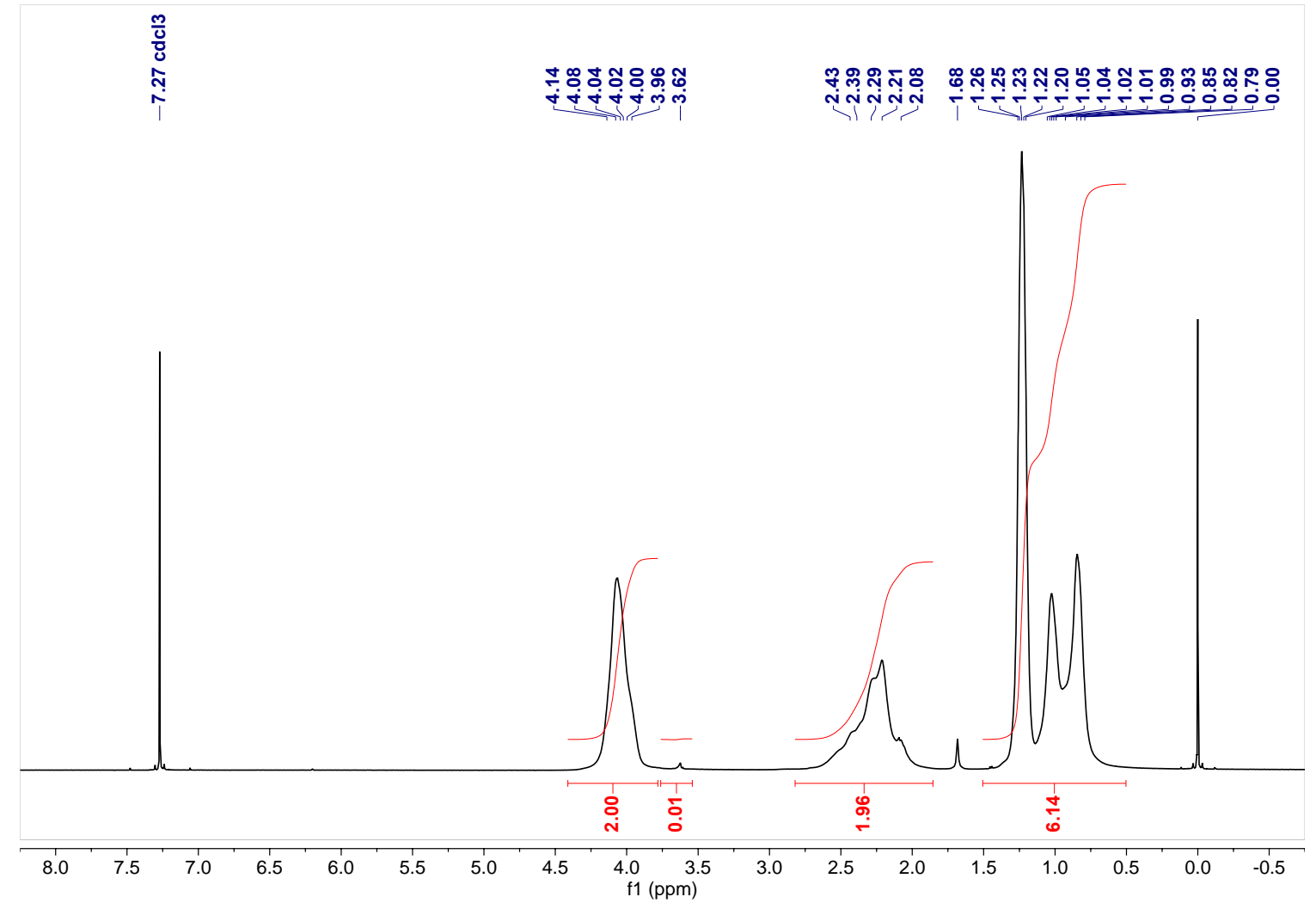

Figure S5. ${ }^{1}$ H NMR spectrum of poly(ethyl crotonate) (Table 5, entry 28).

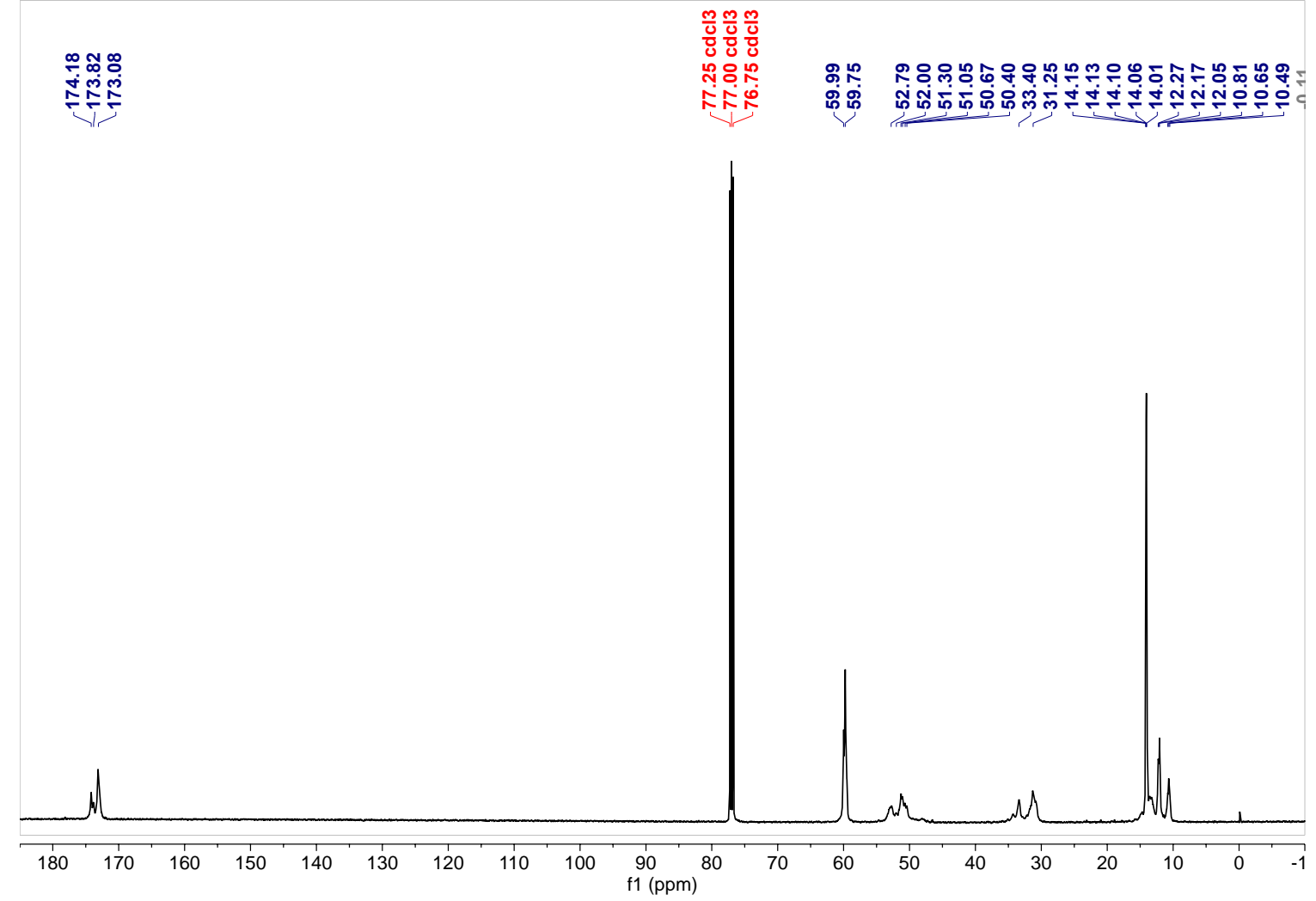

Figure S6. ${ }^{13} \mathrm{C}$ NMR spectrum of poly(ethyl crotonate) (Table 5, entry 28). 


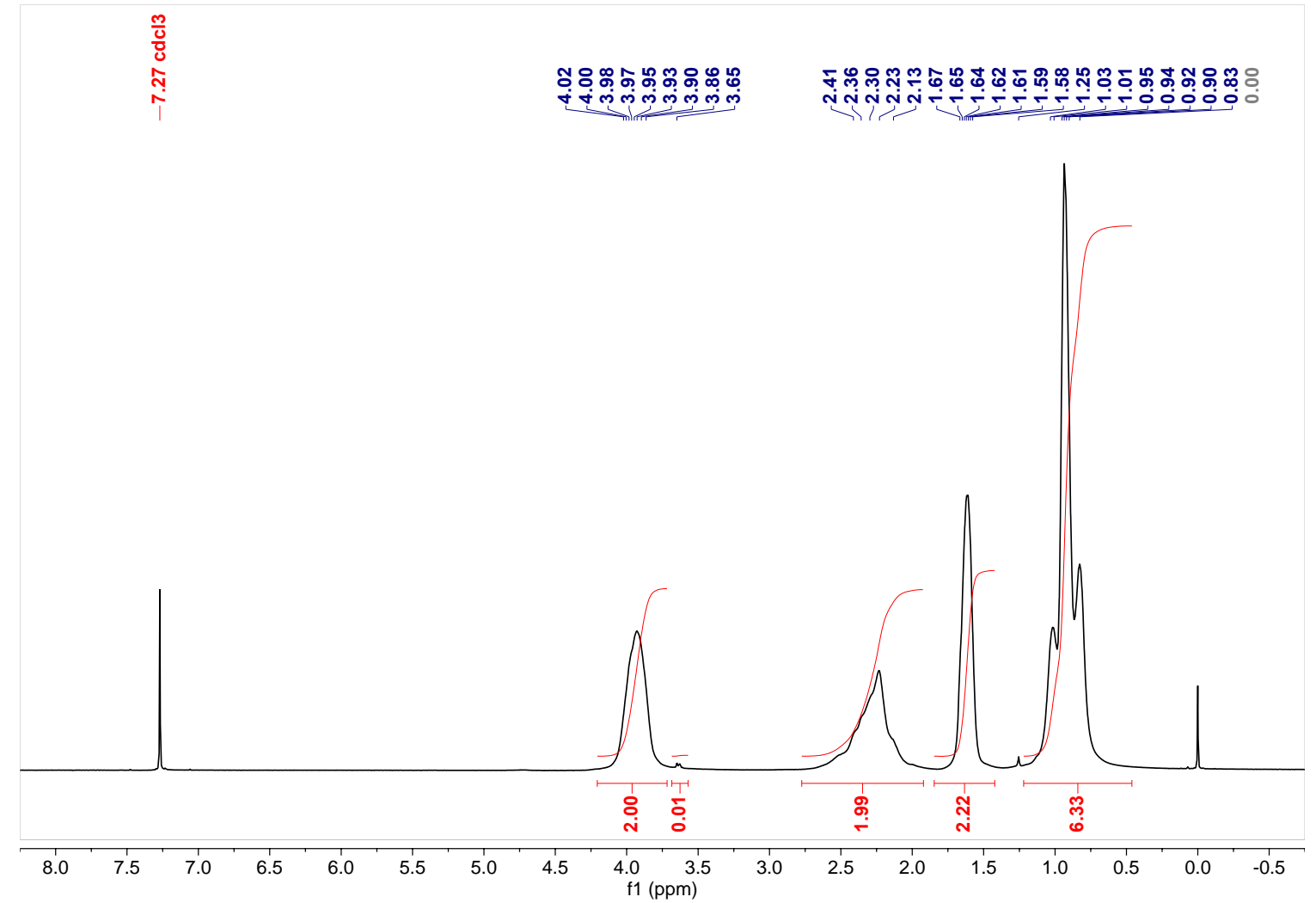

Figure S7. ${ }^{1} \mathrm{H}$ NMR spectrum of poly(n-propyl crotonate) (Table 5, entry 29).

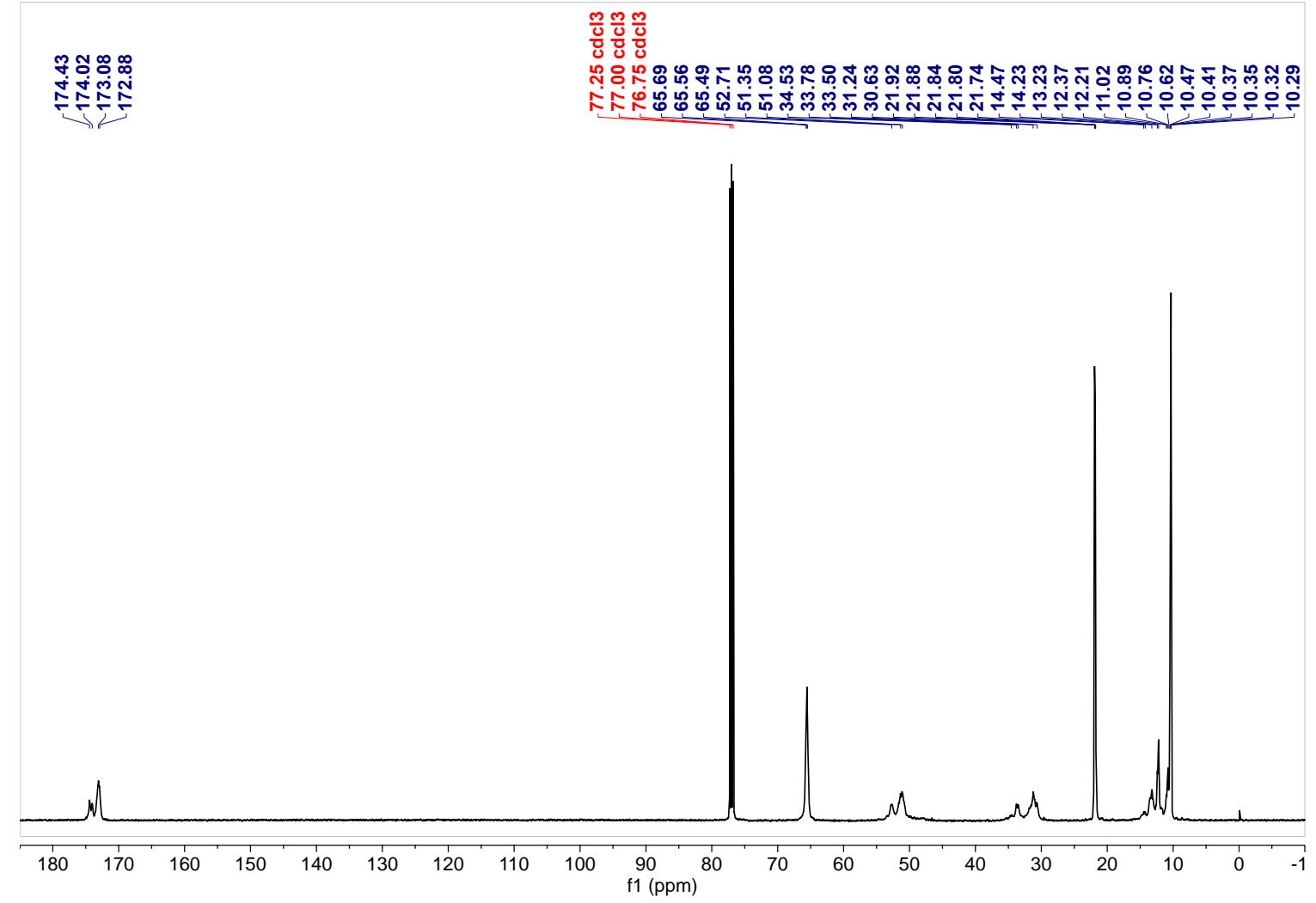

Figure S8. ${ }^{13} \mathrm{C}$ NMR spectrum of poly(n-propyl crotonate) (Table 5, entry 29). 


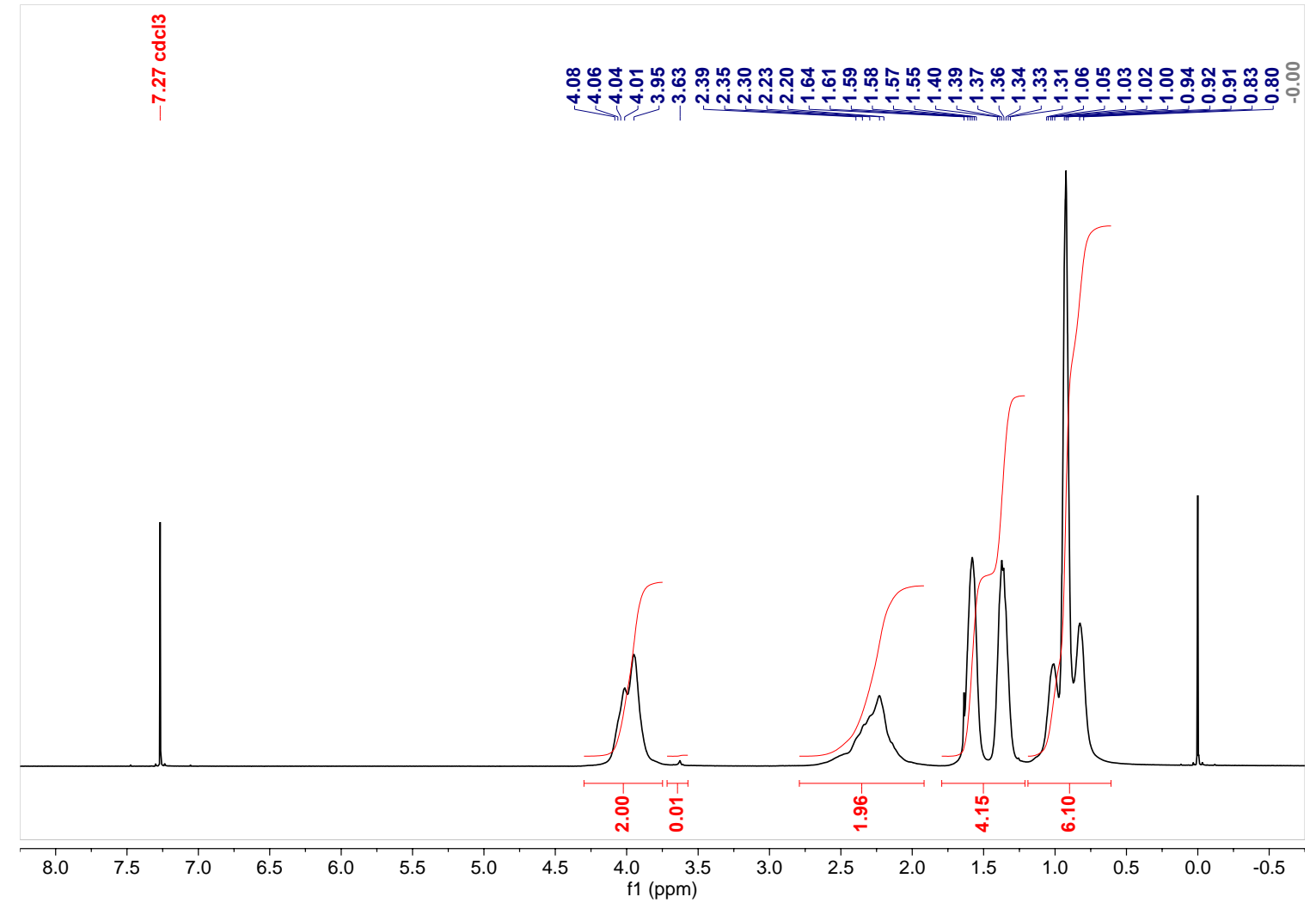

Figure. S9. ${ }^{1} \mathrm{H}$ NMR spectrum of poly( $n$-butyl crotonate) (Table 5, entry 30$)$.

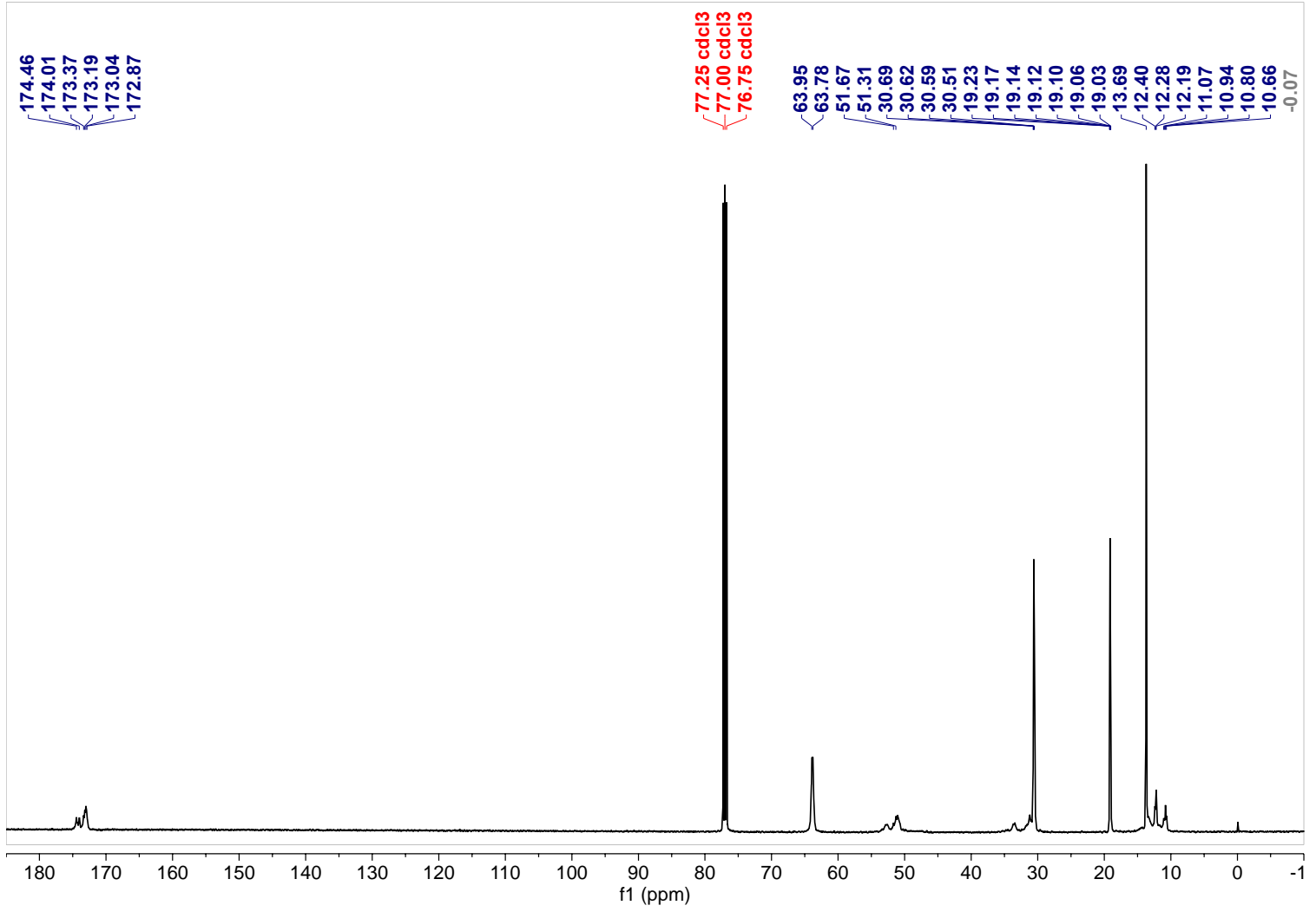

Figure S10. ${ }^{13} \mathrm{C}$ NMR spectrum of poly(n-butyl crotonate) (Table 5, entry 30$)$. 


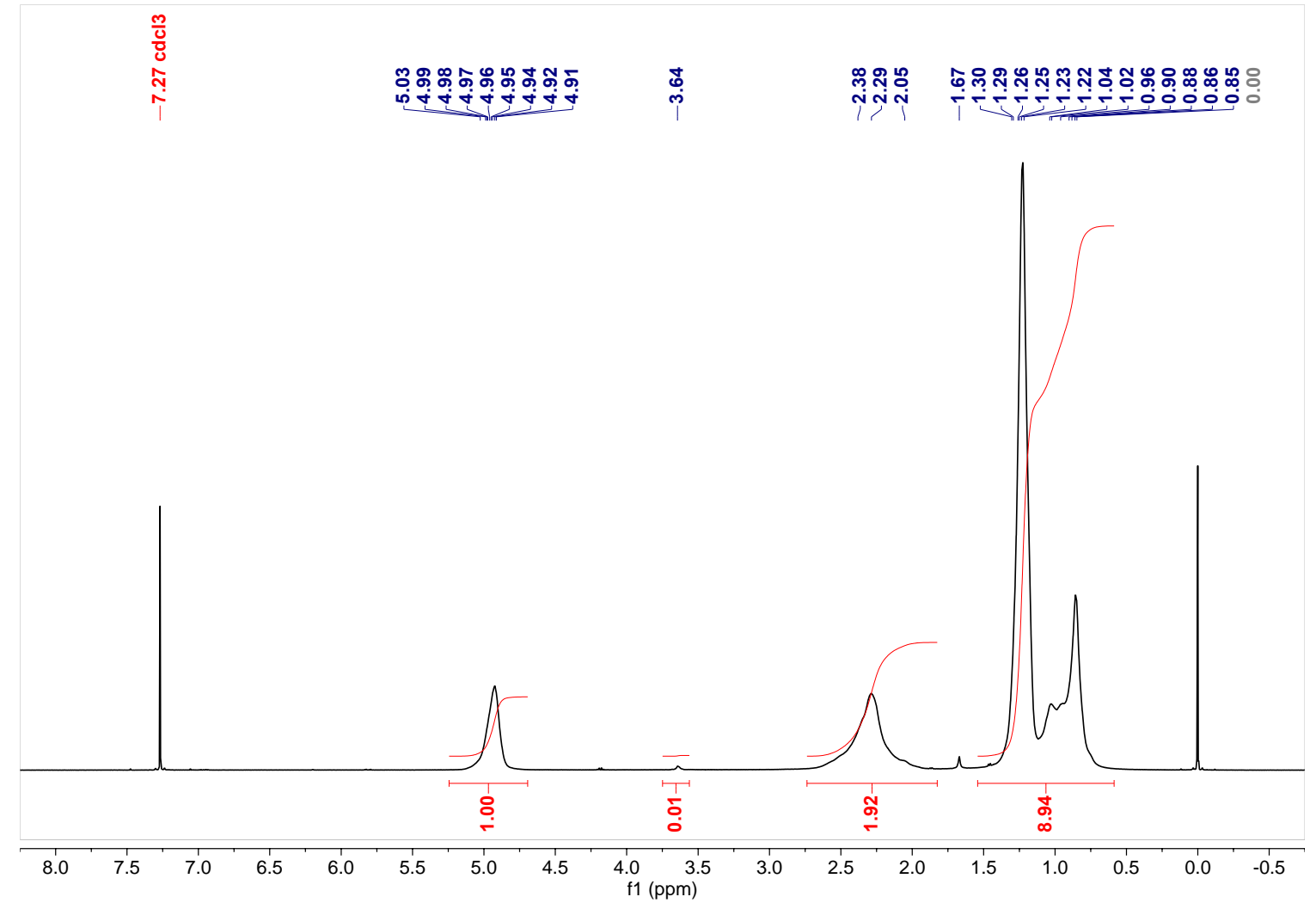

Figure S11. ${ }^{1}$ H NMR spectrum of poly(isopropyl crotonate) (Table 5, entry 31).

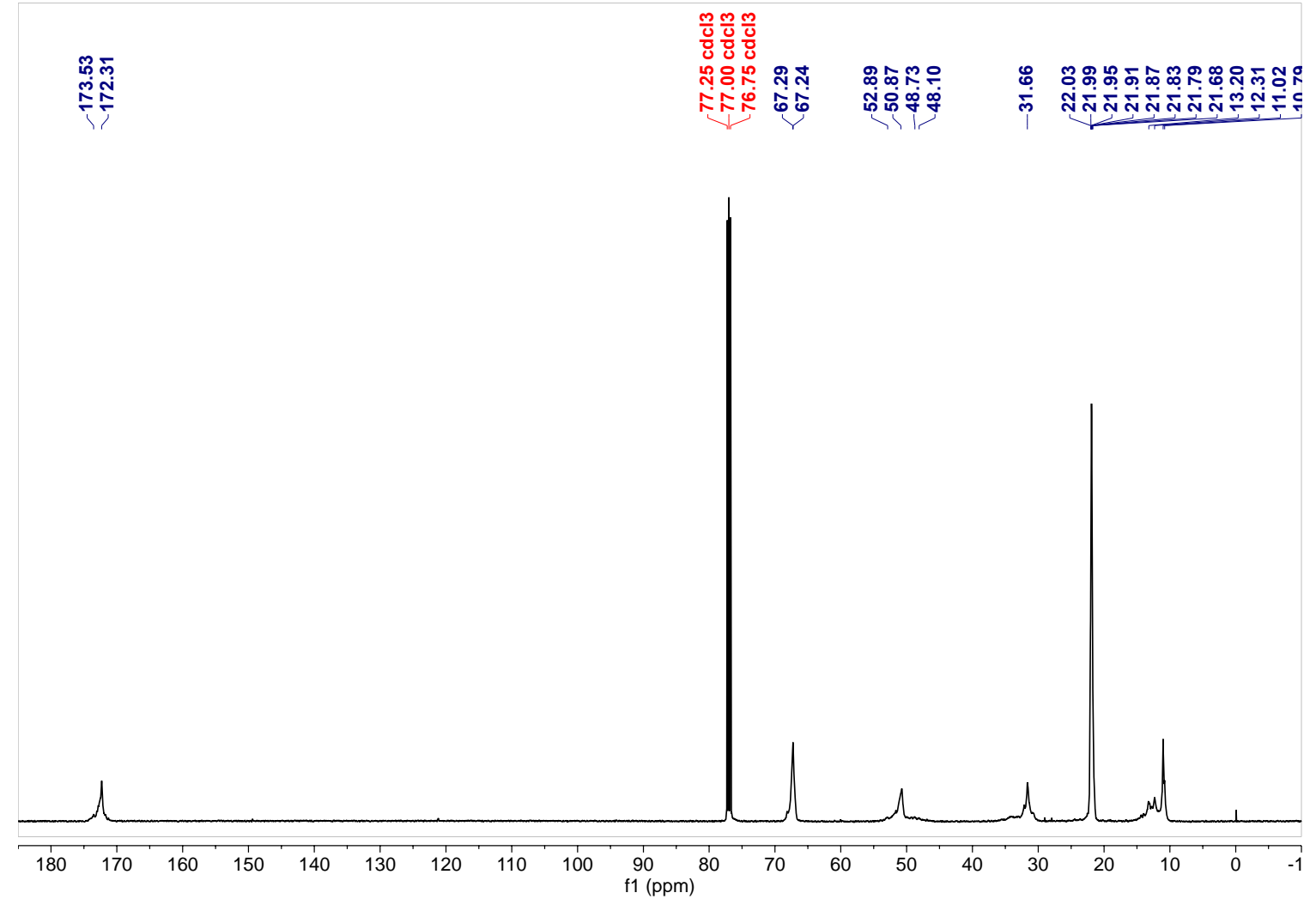

Figure S12. ${ }^{13} \mathrm{C}$ NMR spectrum of poly(isopropyl crotonate) (Table 5, entry 31 ). 


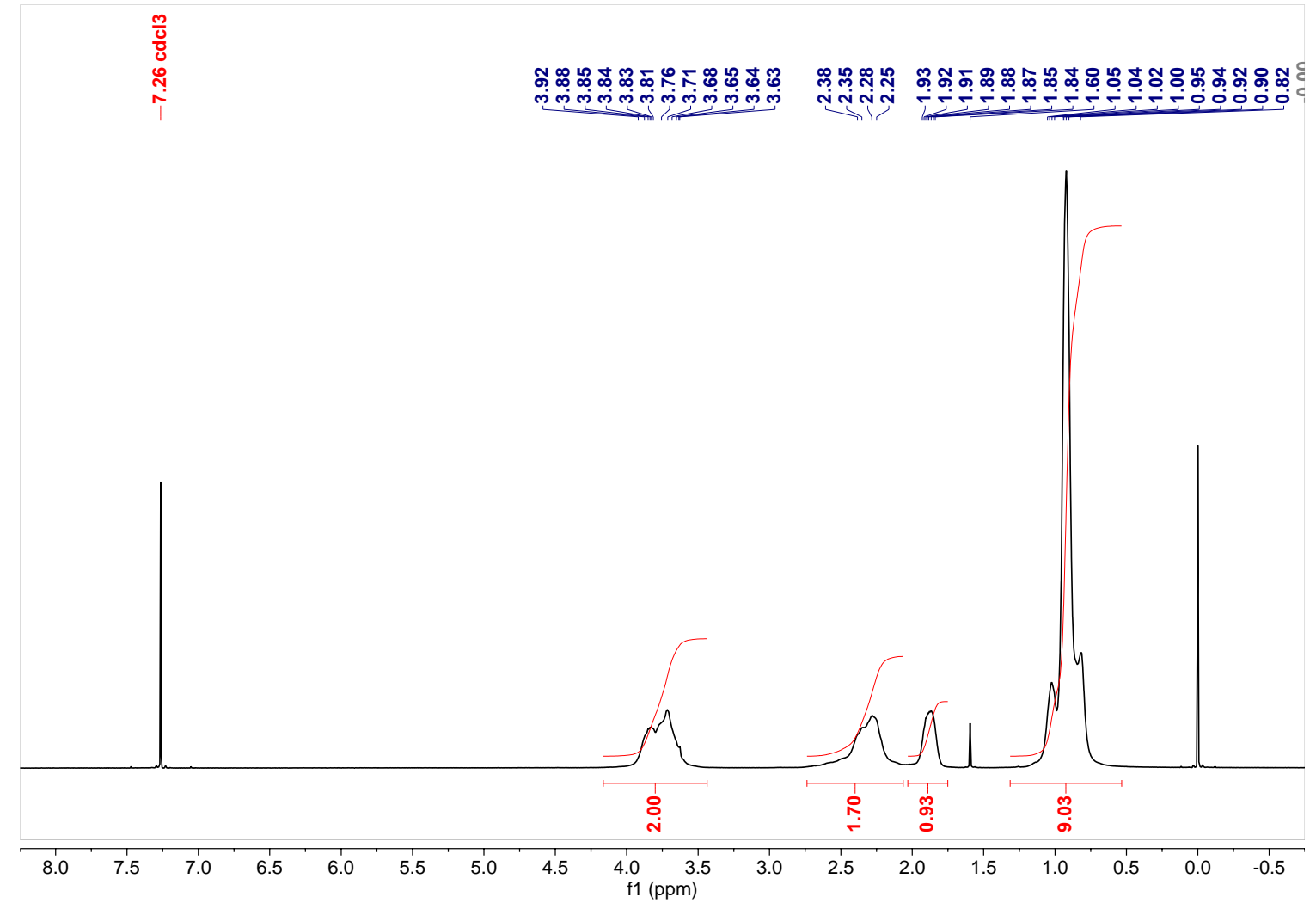

Figure S13. ${ }^{1} \mathrm{H}$ NMR spectrum of poly(isobutyl crotonate) (Table 5, entry 32).

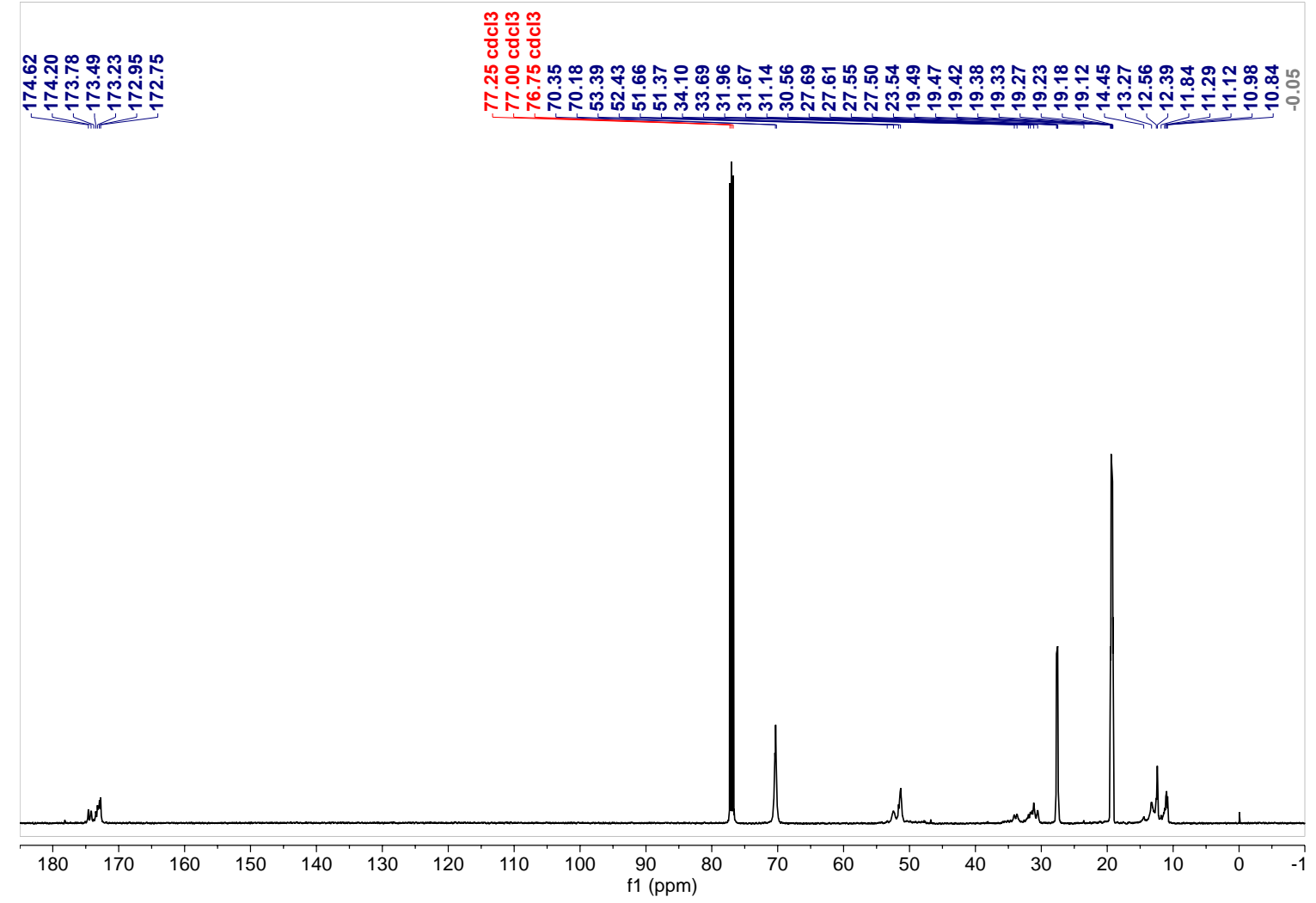

Figure S14. ${ }^{13} \mathrm{C}$ NMR spectrum of poly(isobutyl crotonate) (Table 5, entry 32). 


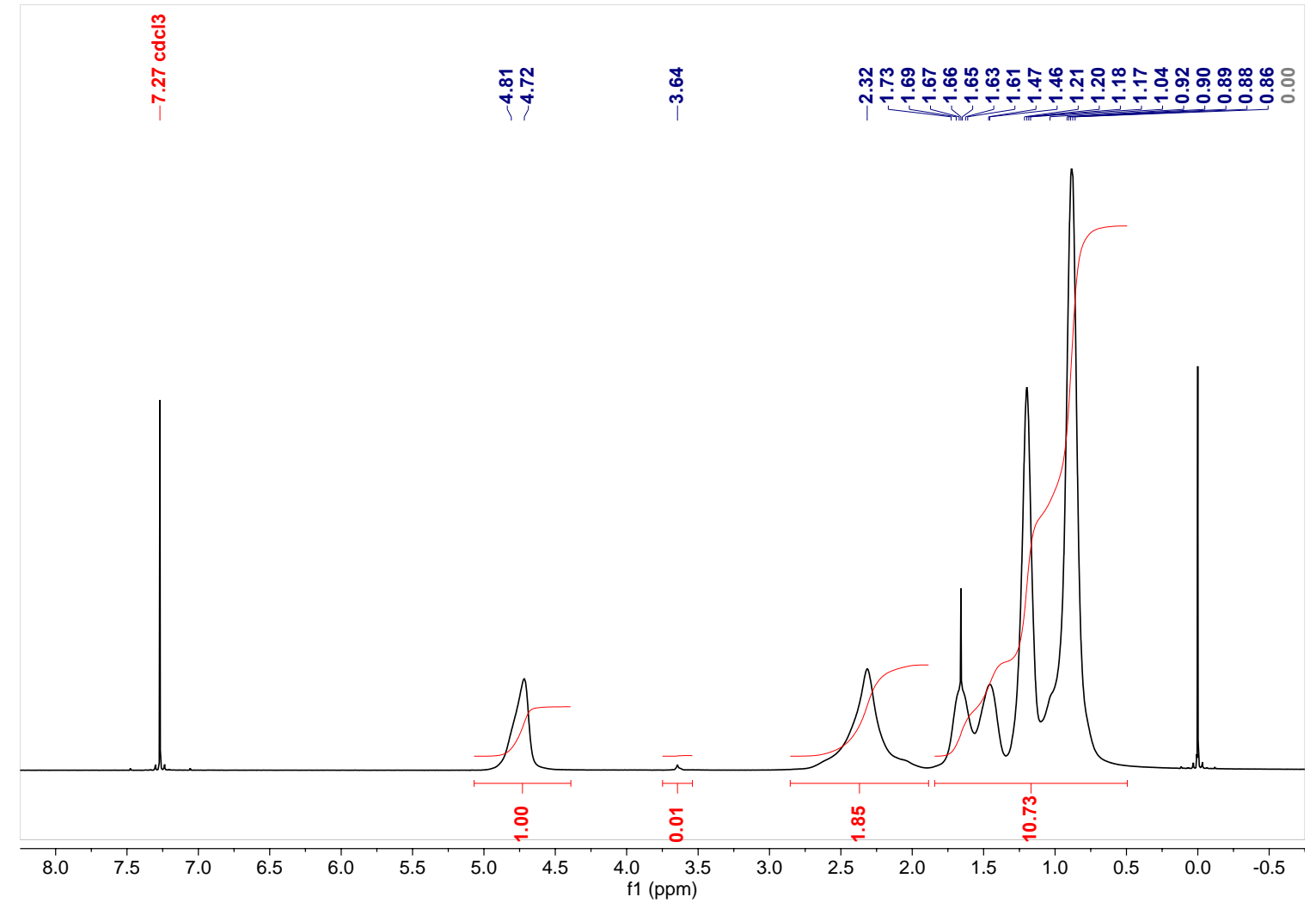

Figure S15. ${ }^{1} \mathrm{H}$ NMR spectrum of poly(sec-butyl crotonate) (Table 5, entry 33).

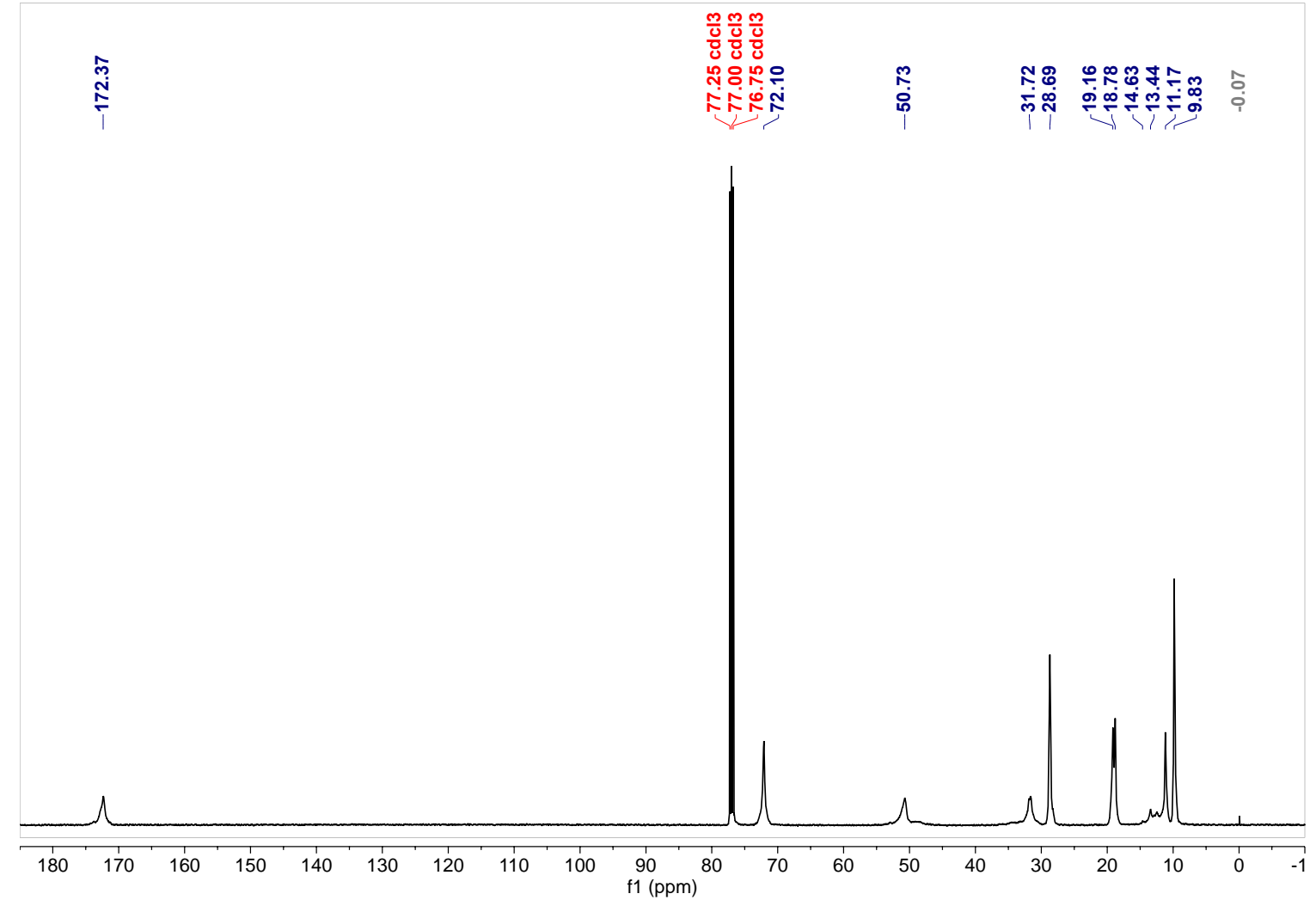

Figure S16. ${ }^{13} \mathrm{C}$ NMR spectrum of poly(sec-butyl crotonate) (Table 5, entry 33). 

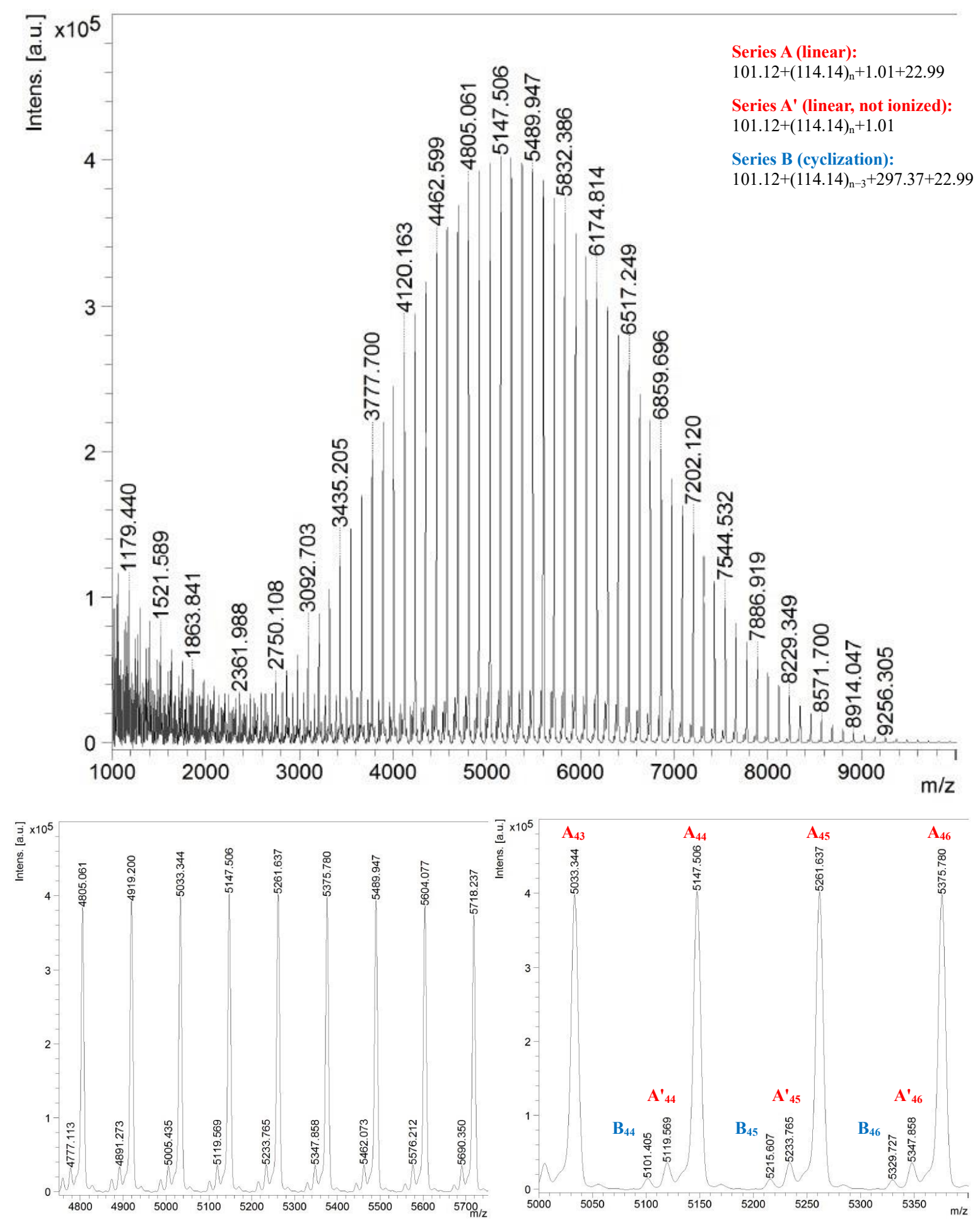

Figure S17. MALDI-TOF-MS spectrum of poly(ethyl crotonate) prepared by GTP at $-40{ }^{\circ} \mathrm{C}$ for $12 \mathrm{~h}$. 

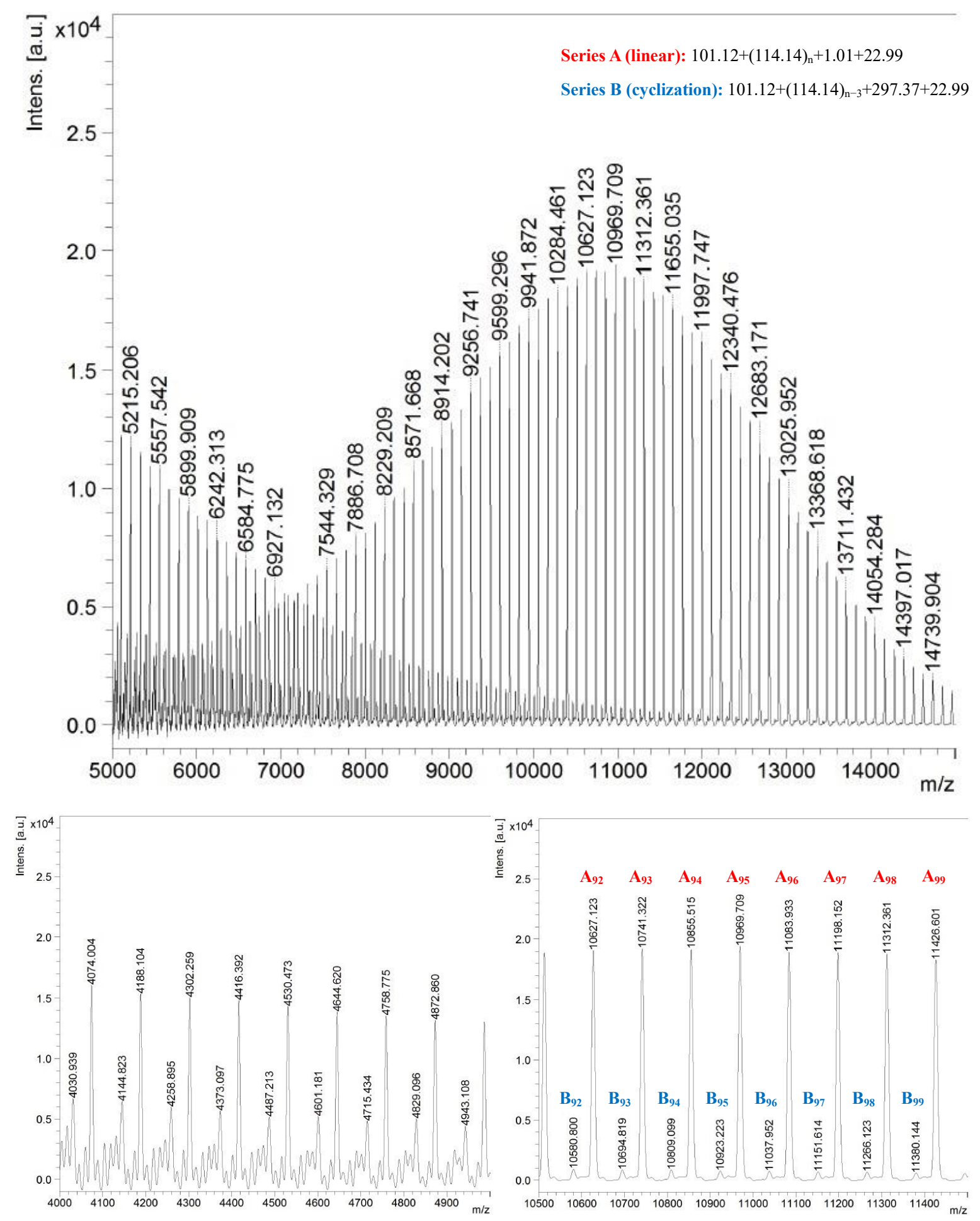

Figure S18. MALDI-TOF-MS spectrum of poly(ethyl crotonate) prepared by GTP at $-40{ }^{\circ} \mathrm{C}$ for $24 \mathrm{~h}$ (Table 2, entry 3 ). 

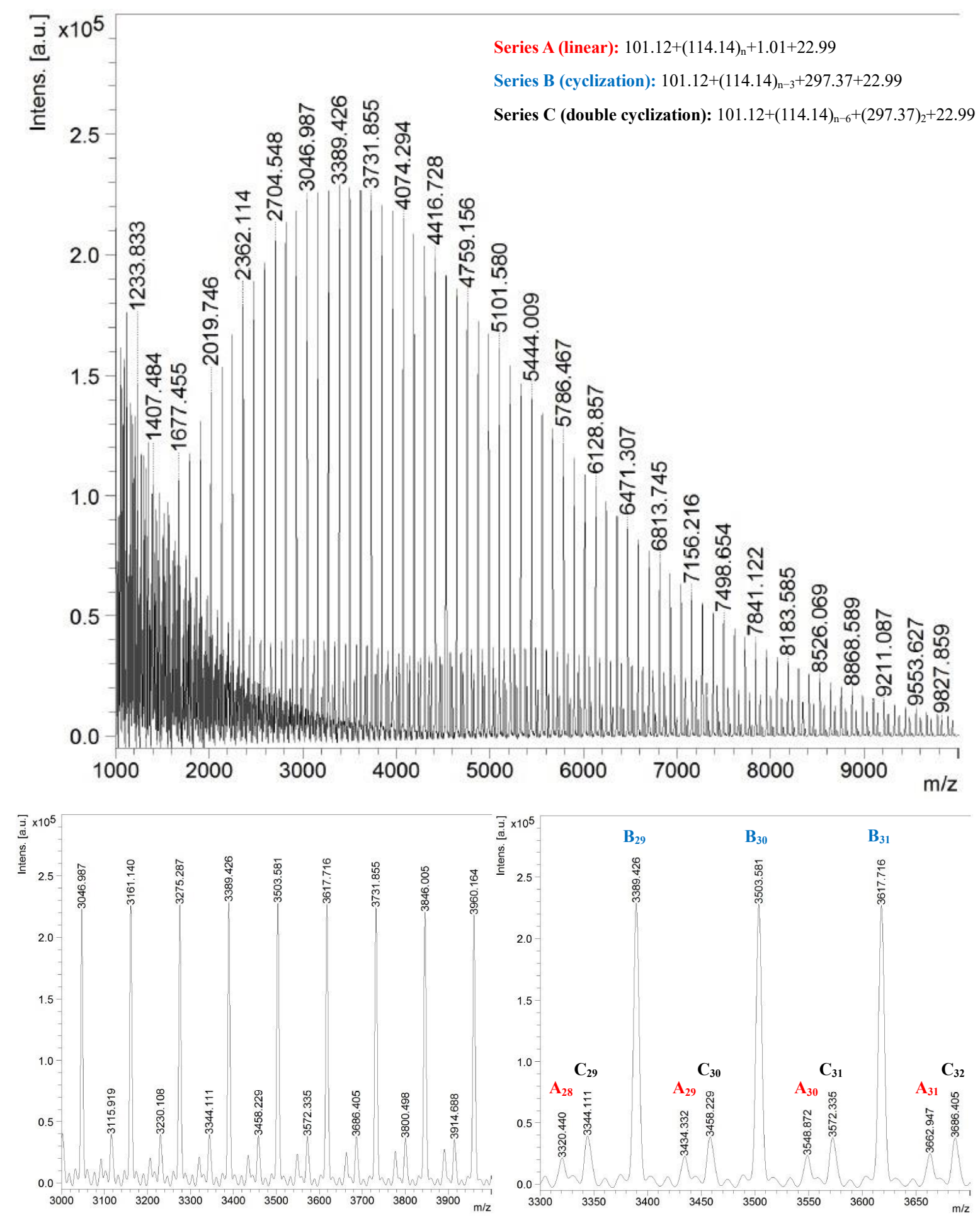

Figure S19. MALDI-TOF-MS spectrum of poly(ethyl crotonate) prepared by GTP at $20^{\circ} \mathrm{C}$ for $24 \mathrm{~h}$ (Table 2 , entry 8 ). 

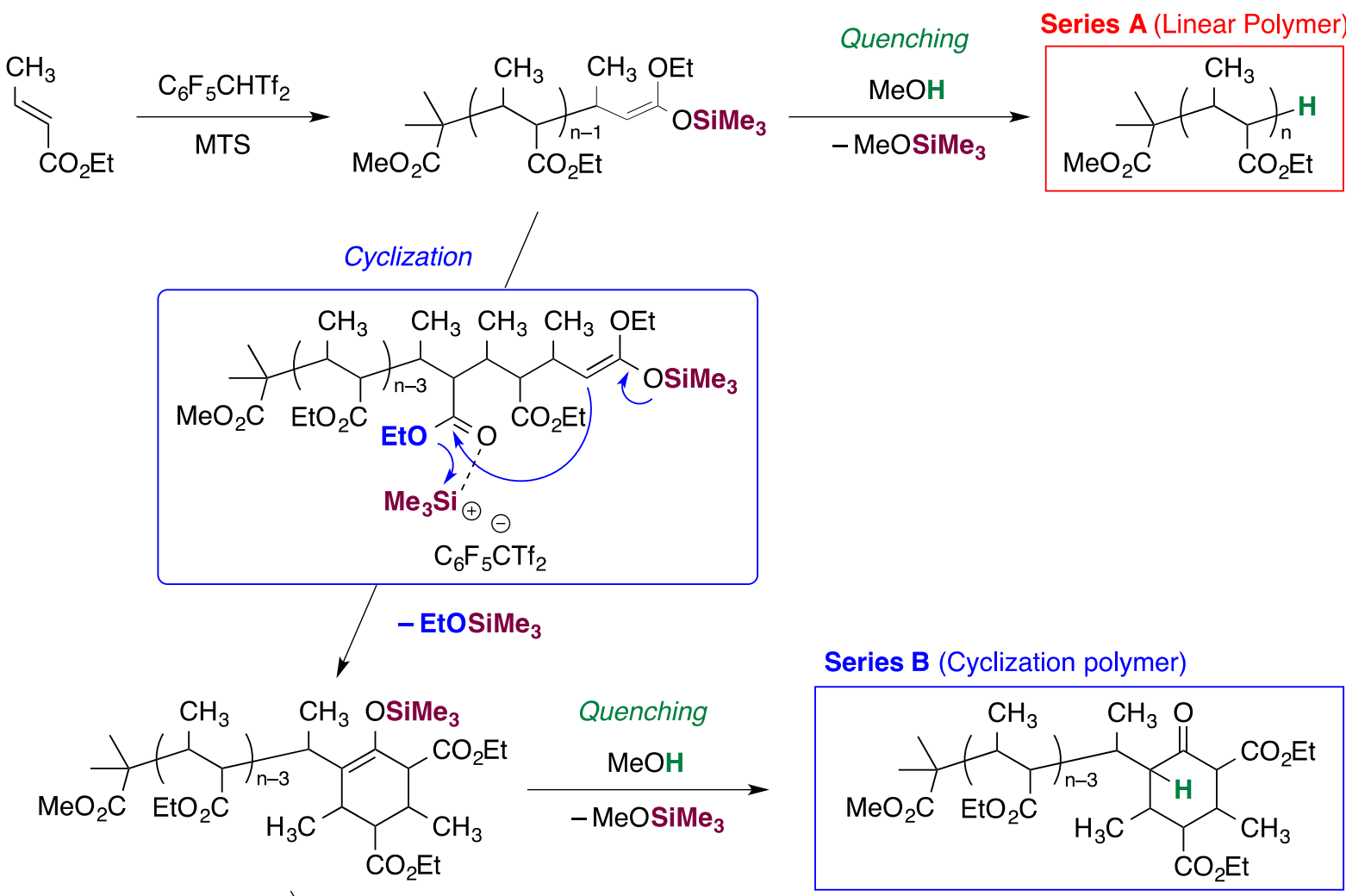

Series B (Cyclization polymer)
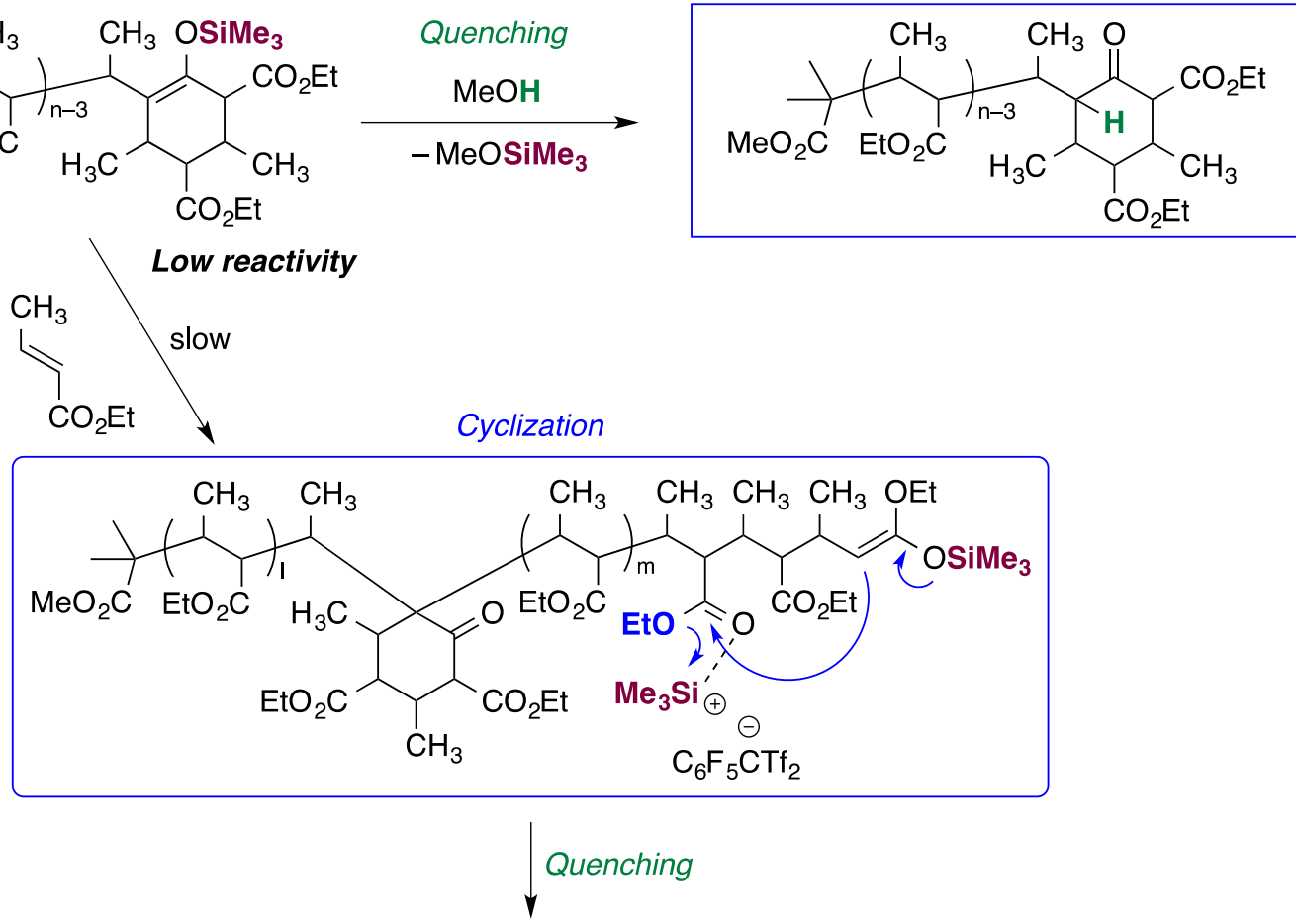

Series C (Double Cyclization Polymer)

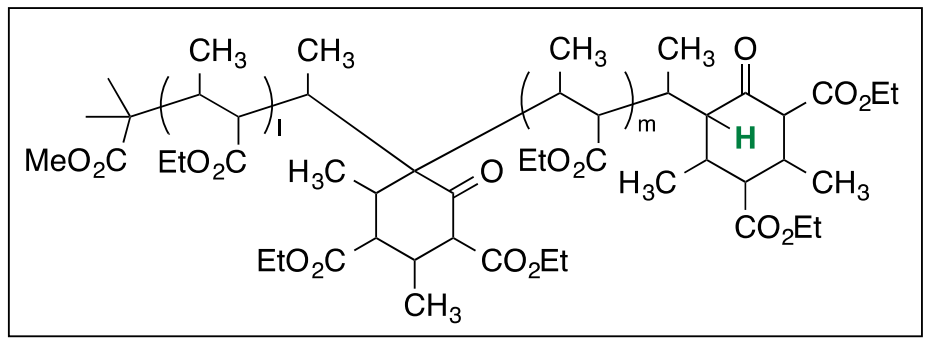

Scheme S1. The cyclization reaction of propagating chain-end groups in the GTP of ethyl crotonate. 


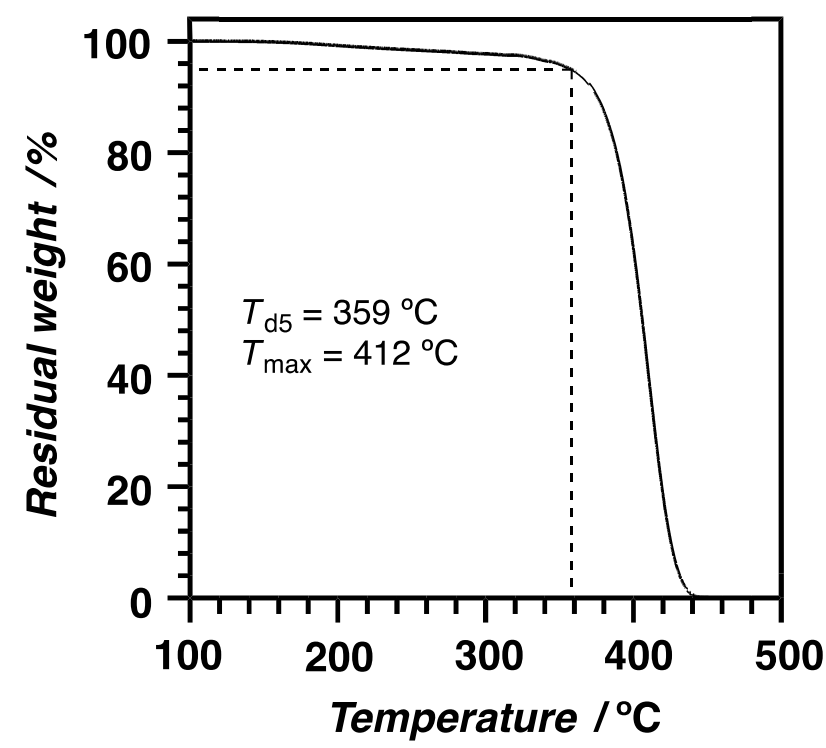

Figure S20. TGA curve of poly(methyl crotonate) (Table 5, entry 27).

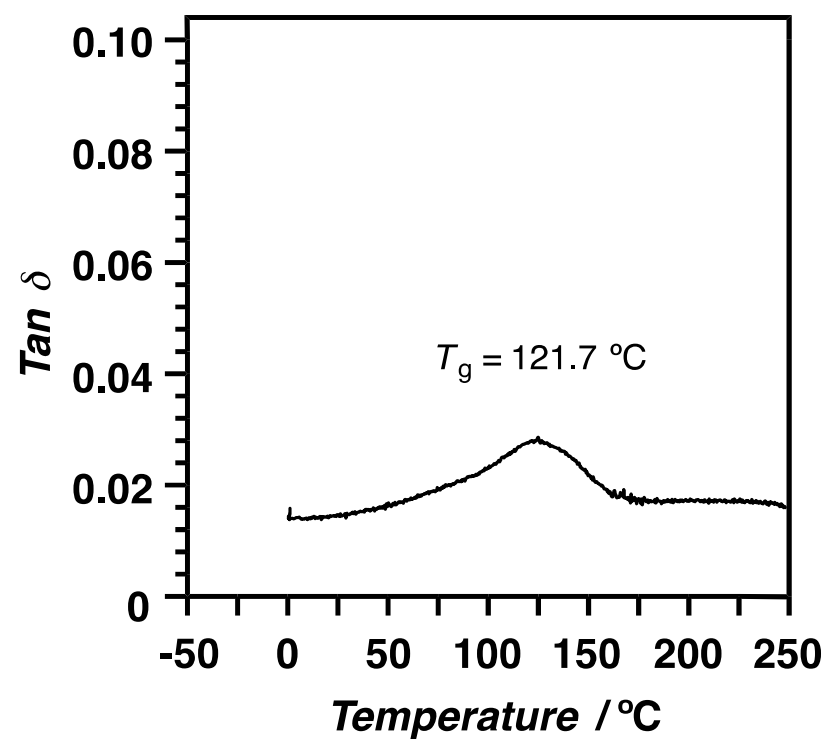

Figure S21. DMA curve of poly(methyl crotonate) (Table 5, entry 27). 


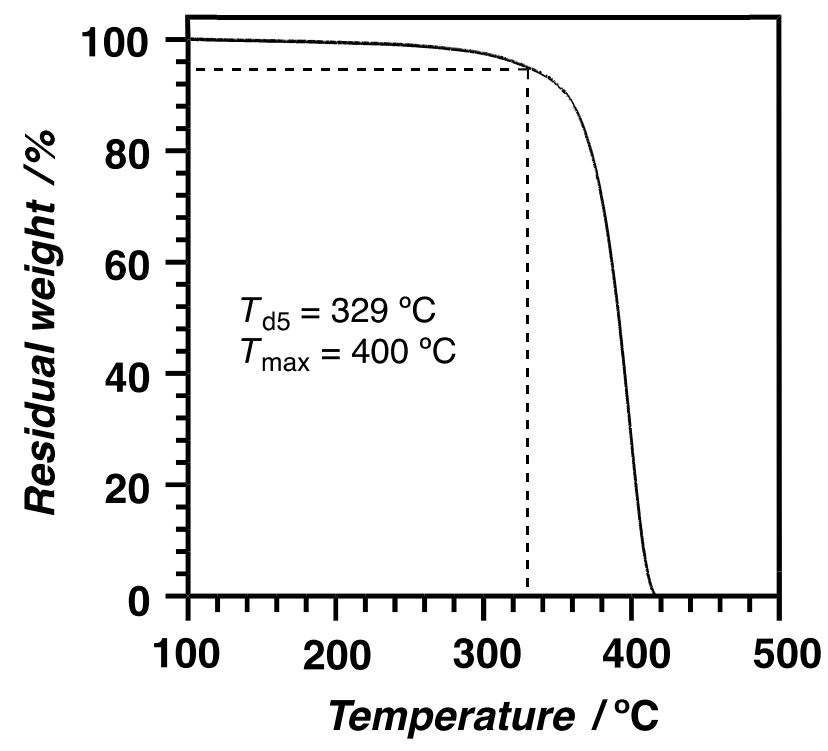

Figure S22. TGA curve of poly(ethyl crotonate) (Table 5, entry 28).

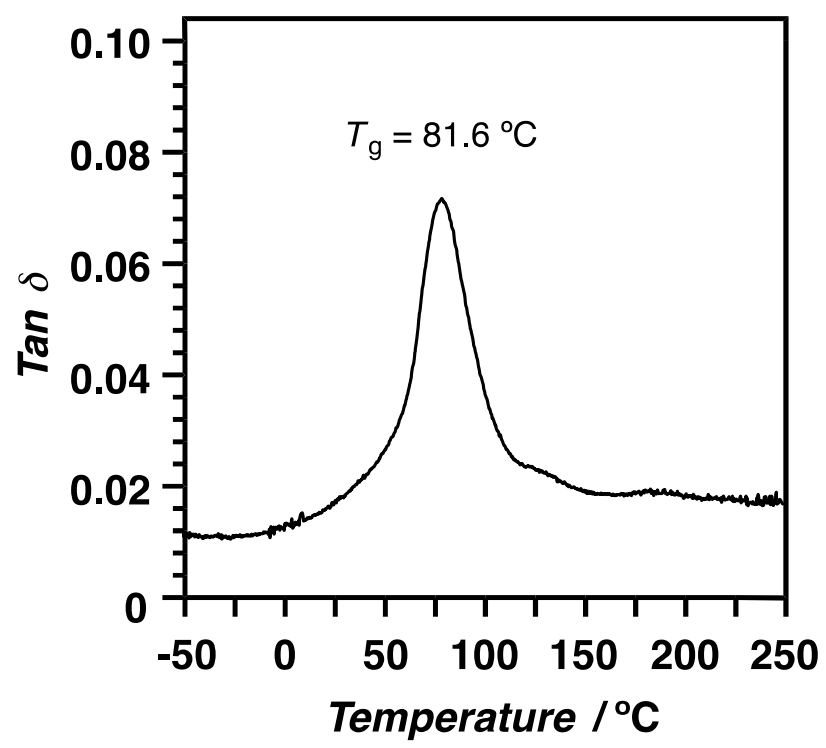

Figure S23. DMA curve of poly(ethyl crotonate) (Table 5, entry 28). 


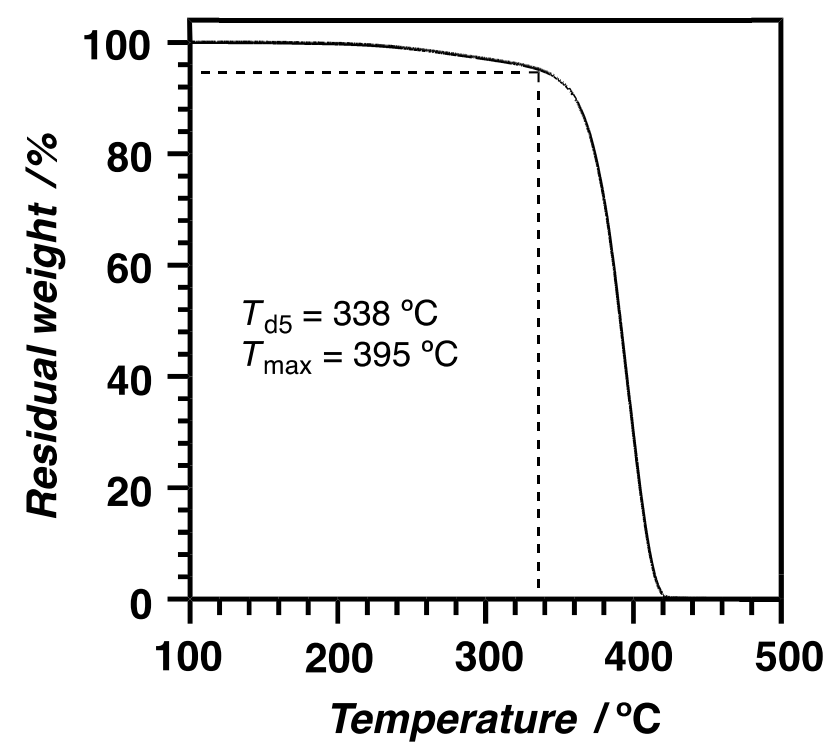

Figure S24. TGA curve of poly(n-propyl crotonate) (Table 4, entry 20).

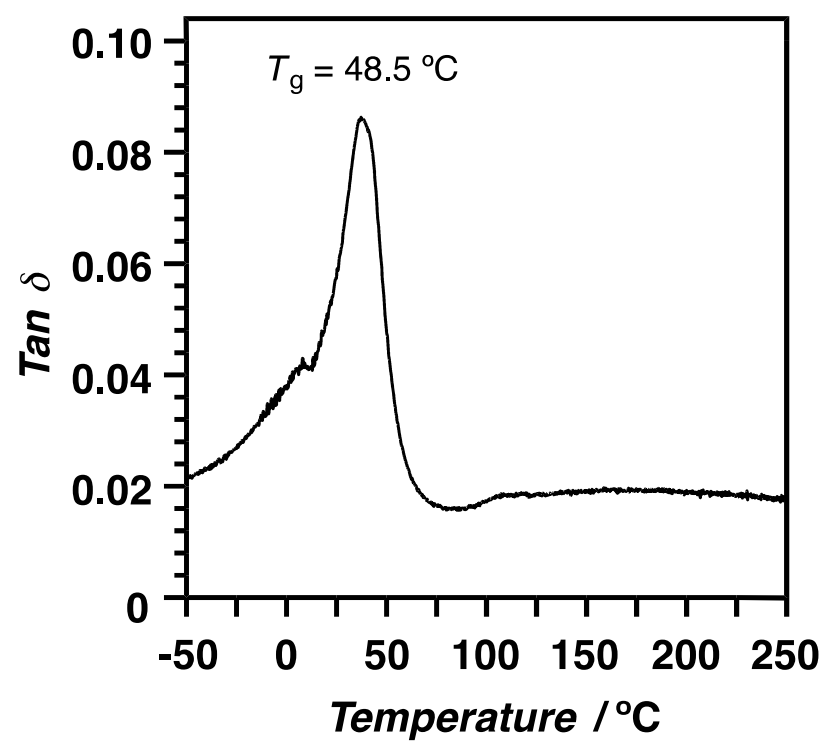

Figure S25. DMA curve of poly(n-propyl crotonate) (Table 4, entry 20). 


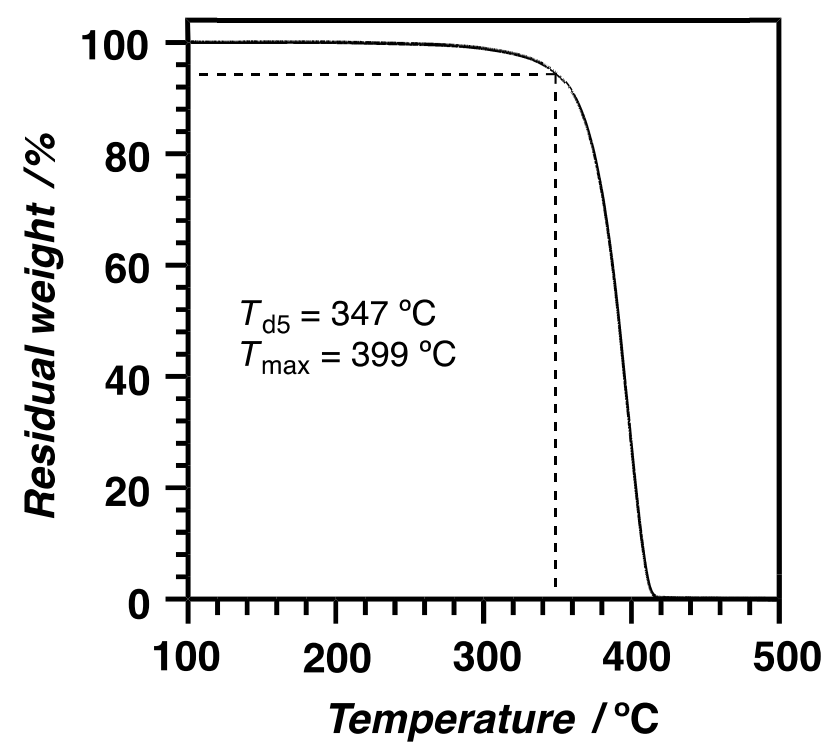

Figure S26. TGA curve of $\operatorname{poly}(n$-butyl crotonate) (Table 5, entry 30$)$.

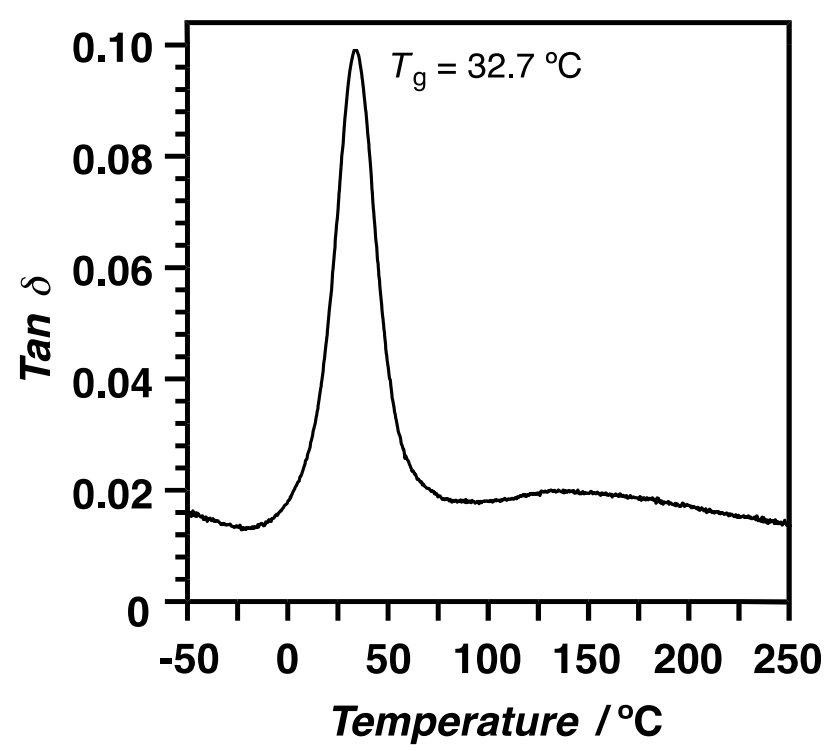

Figure S27. DMA curve of poly( $n$-butyl crotonate) (Table 5, entry 30). 


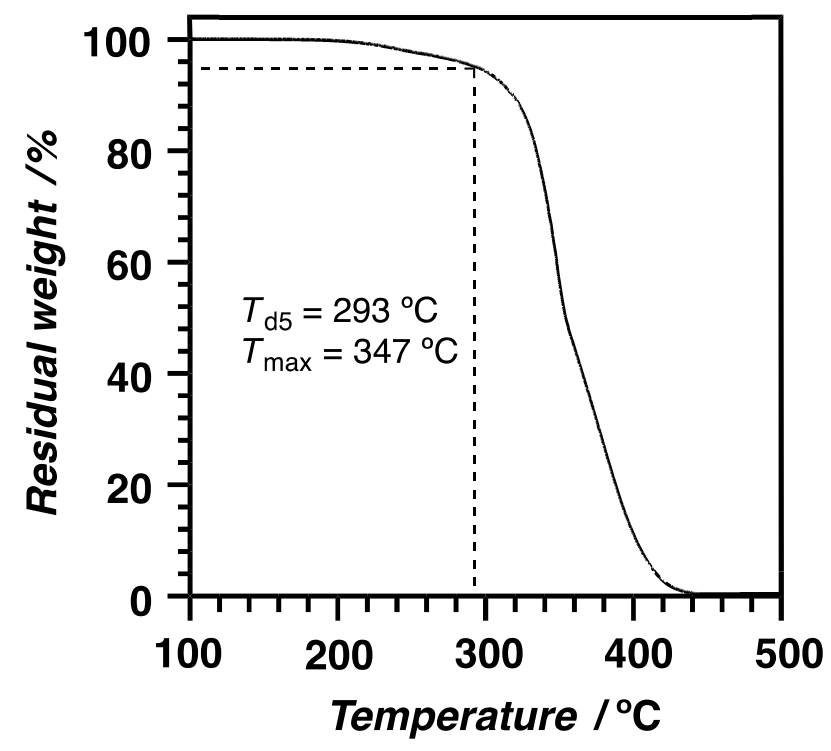

Figure S28. TGA curve of poly(isopropyl crotonate) (Table 5, entry 31).

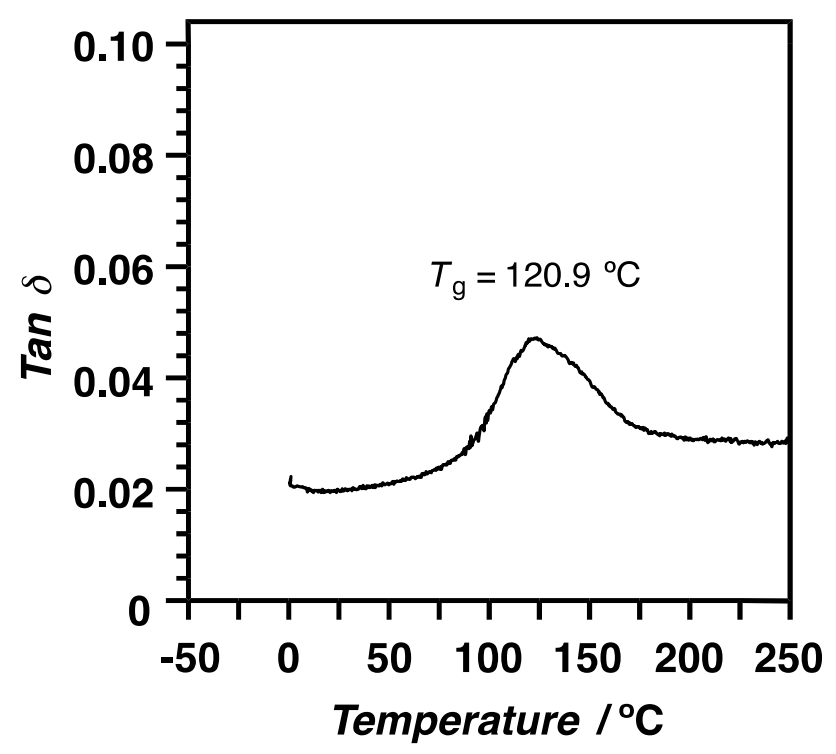

Figure S29. DMA curve of poly(isopropyl crotonate) (Table 5, entry 31). 


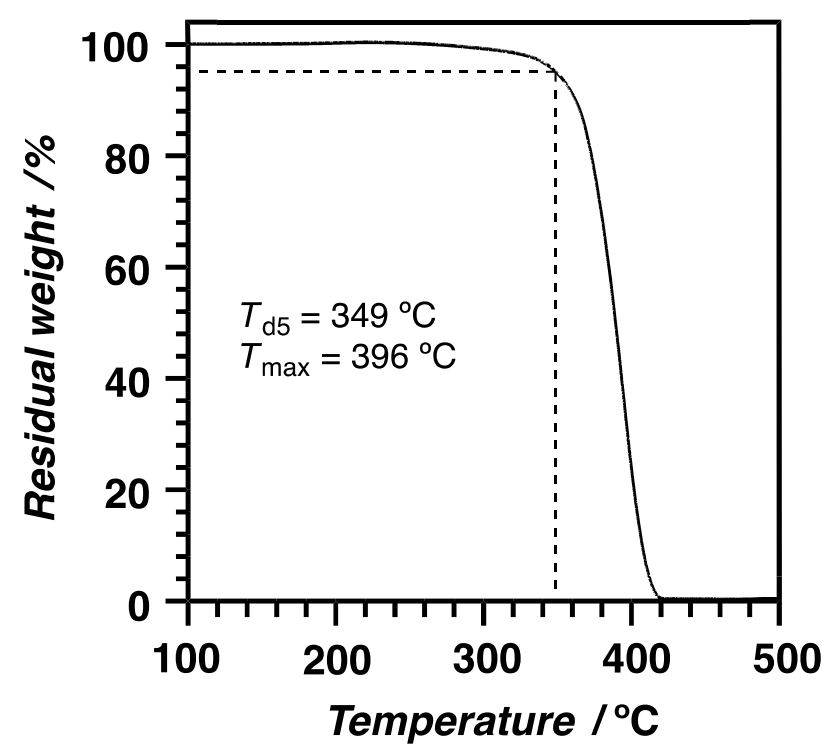

Figure S30. TGA curve of poly(isobutyl crotonate) (Table 5, entry 32).

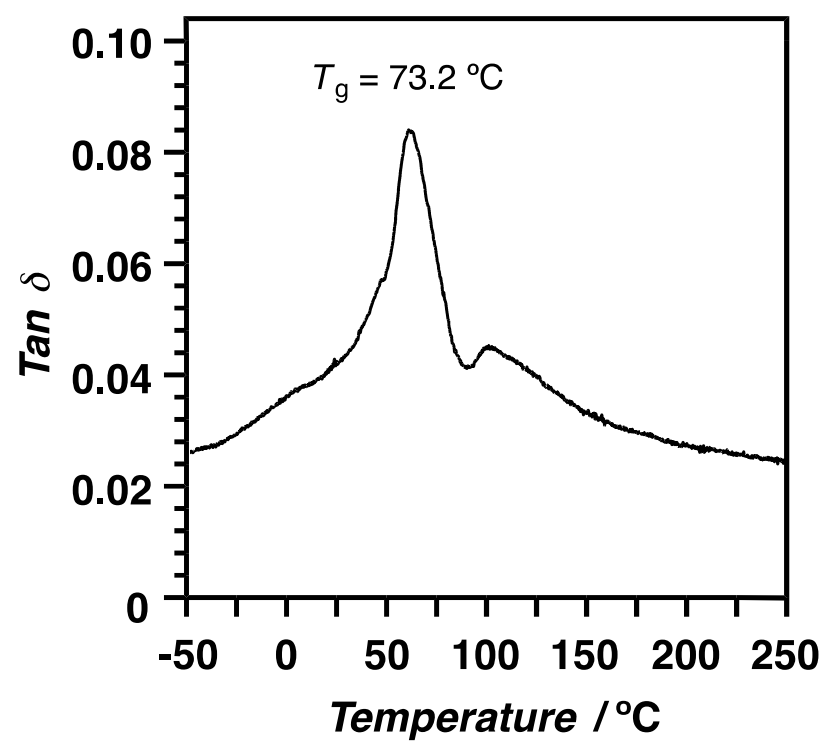

Figure S31. DMA curve of poly(isobutyl crotonate) (Table 5, entry 32). 


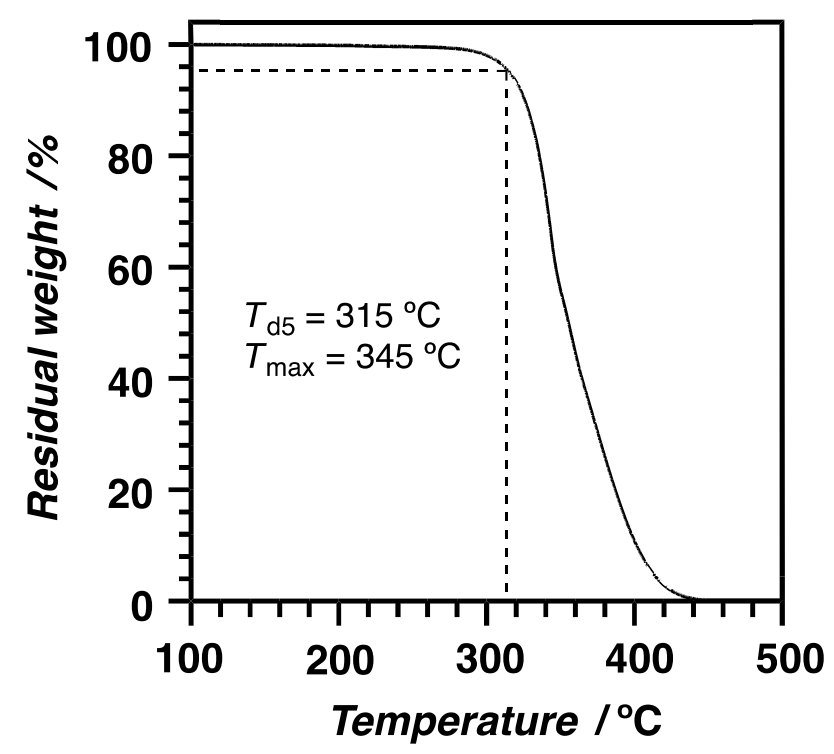

Figure S32. TGA curve of poly(sec-butyl crotonate) (Table 5, entry 33).

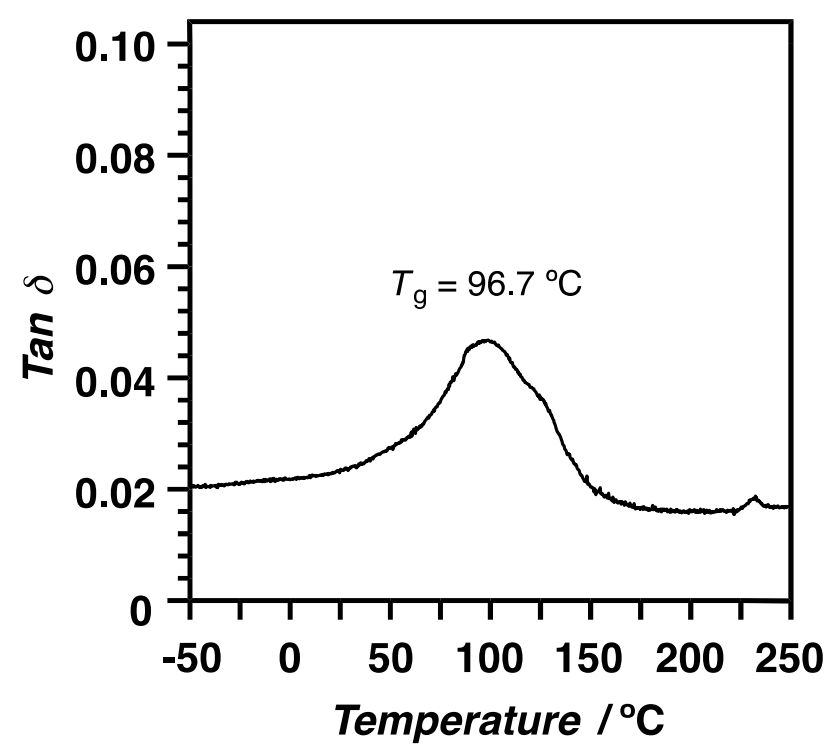

Figure S33. TGA curve of poly(sec-butyl crotonate) (Table 5, entry 33). 


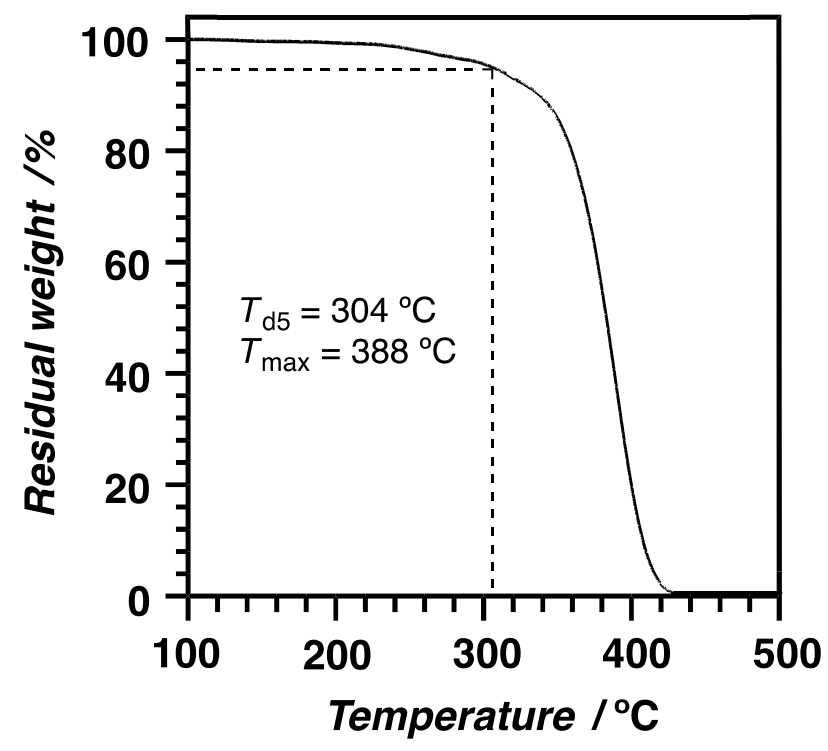

Figure S34. TGA curve of PMMA prepared by GTP.

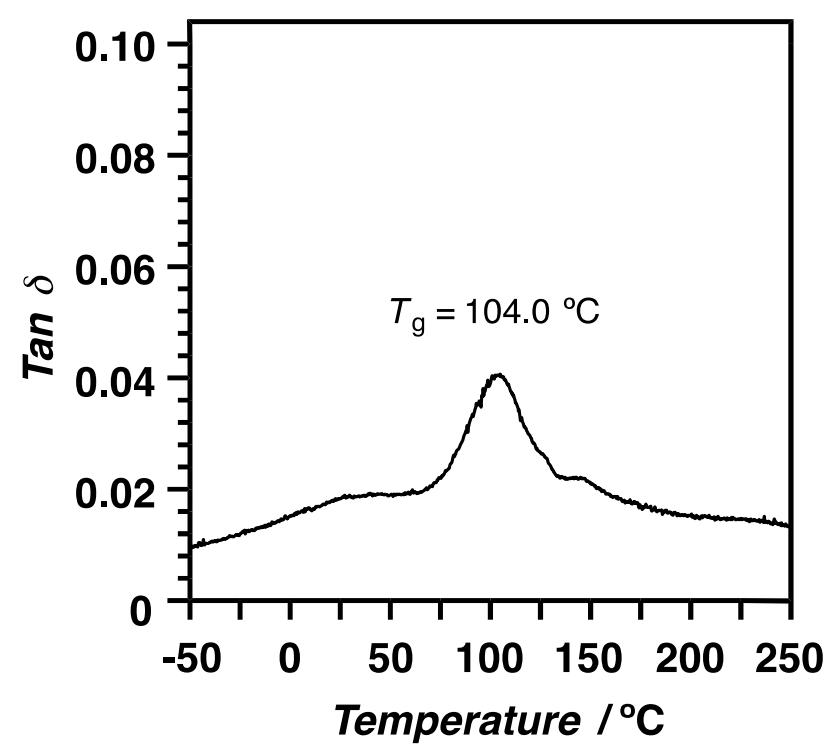

Figure S35. DMA curve of PMMA prepared by GTP. 
(a) $\mathrm{PMeCr}$

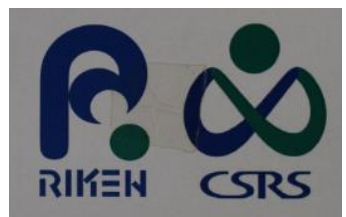

(e) PiPrCr

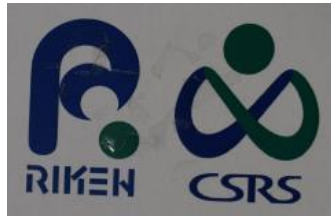

(b) PEtCr

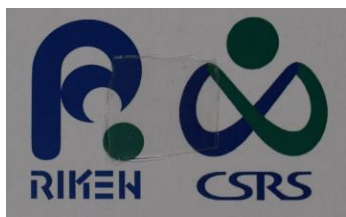

(f) $\mathrm{P}^{i} \mathrm{BuCr}$

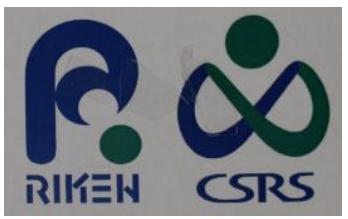

(c) $\mathrm{P}^{n} \mathrm{PrCr}$

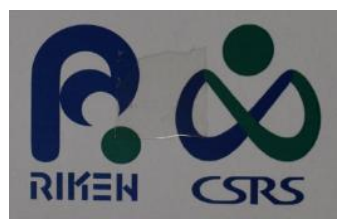

(d) $\mathrm{P}^{n} \mathrm{BuCr}$

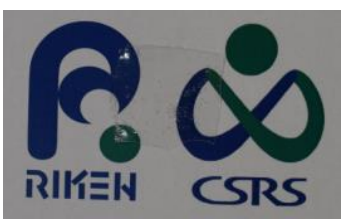

Figure S36. The pressed films of polymers
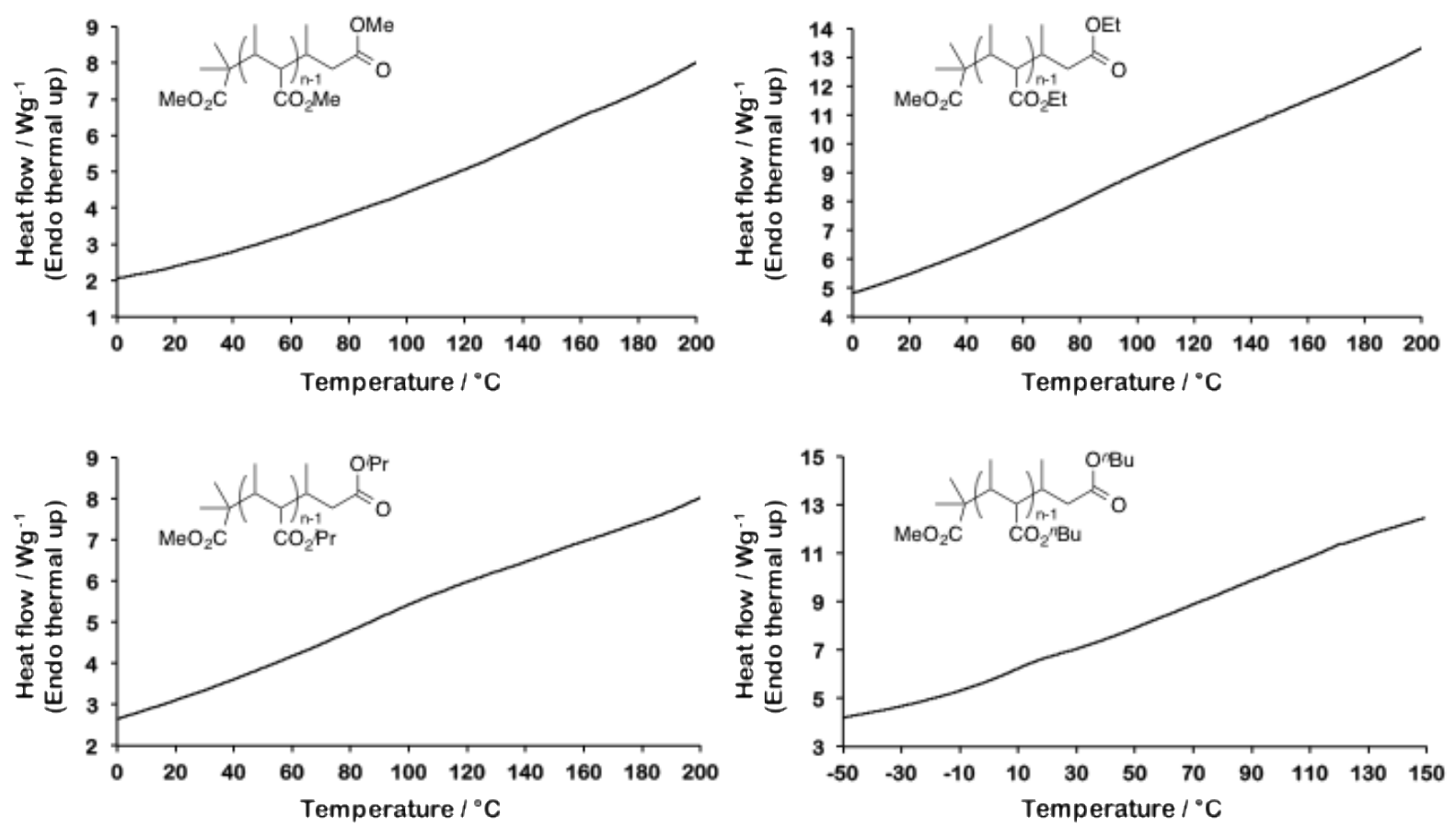

Figure S37. The DSC curves of polymers. 
Table S1. The organic acid-catalyzed GTP of various crotonates ${ }^{a}$

\begin{tabular}{llllll}
\hline $\mathrm{R}$ & $\begin{array}{l}\text { time } \\
\mathrm{h}\end{array}$ & $\begin{array}{l}\text { conv. }^{b} \\
\%\end{array}$ & $\begin{array}{l}\text { yield }^{c} \\
\%\end{array}$ & $\begin{array}{l}M_{\mathrm{n}}{ }^{d} \\
\mathrm{~kg} / \mathrm{mol}\end{array}$ & $M_{\mathrm{w}} / M_{\mathrm{n}}{ }^{d}$ \\
\hline methyl & 96 & $>99$ & $>99$ & 29.7 & 1.19 \\
ethyl & 48 & $>99$ & $>99$ & 22.8 & 1.22 \\
$n$-propyl & 48 & $>99$ & $>99$ & 22.8 & 1.11 \\
$n$-butyl & 72 & $>99$ & 99 & 26.7 & 1.12 \\
isopropyl & 120 & $>99$ & 98 & 22.6 & 1.11 \\
isobutyl & 144 & $>99$ & $>99$ & 33.4 & 1.14 \\
sec-butyl & 96 & $>99$ & $>99$ & 22.7 & 1.13 \\
\hline
\end{tabular}

${ }^{a}$ Monomer (M) is alkyl crotonate ( $\left.\mathrm{RCr}\right)$, initiator (I) is 1-methoxy-1-trimethylsilyloxy-2-methyl-1-propene (MTS), catalyst is $\mathrm{C}_{6} \mathrm{~F}_{5} \mathrm{CHTf}_{2}, \mathrm{M}=50.0 \mathrm{mmol}, \mathrm{I}=0.50 \mathrm{mmol}$, catalyst $=0.050 \mathrm{mmol}, \mathrm{CH}_{2} \mathrm{Cl}_{2}=25 \mathrm{~mL}$, $[\mathrm{M}]_{0} /[\mathrm{I}]_{0} /[\mathrm{Cat} .]_{0}=100 / 1 / 0.1$, and $[\mathrm{M}]_{0}=$ ca. $1.6 \mathrm{M} .{ }^{b}$ Calculated by the weight of crude sample after removed monomer. ${ }^{c}$ The obtained polymer was purified by reprecipitation. ${ }^{d}$ Determined by $\mathrm{SEC}$ in $\mathrm{CHCl}_{3}$ using polystyrene standards with an RI detector. 